\title{
Hydraulic Characteristics of Transporting a Piped Carriage in a Horizontal Pipe Based on the Bidirectional Fluid-Structure Interaction
}

\author{
Chunjin Zhang, ${ }^{1}$ Xihuan Sun $\left(1,{ }^{1,2}\right.$ Yongye Li, ${ }^{1}$ Xueqin Zhang, ${ }^{3}$ \\ Xuelan Zhang, ${ }^{1}$ Xiaoni Yang, ${ }^{1,4}$ and Fei $\mathrm{Li}^{1}$ \\ ${ }^{1}$ School of Hydro Science \& Engineering, Taiyuan University of Technology, Taiyuan 030024, China \\ ${ }^{2}$ Jinzhong University, Jinzhong 030600, China \\ ${ }^{3}$ Zhangqiu Yellow River Bureau, Jinan 250200, China \\ ${ }^{4}$ Polytechnic Institute Taiyuan University of Technology, Xiaoyi 032300, China
}

Correspondence should be addressed to Xihuan Sun; sunxihuan@tyut.edu.cn

Received 26 April 2018; Accepted 8 July 2018; Published 10 October 2018

Academic Editor: Eric Feulvarch

Copyright (c) 2018 Chunjin Zhang et al. This is an open access article distributed under the Creative Commons Attribution License, which permits unrestricted use, distribution, and reproduction in any medium, provided the original work is properly cited.

Energy shortage restricts the rapid development of the global transport industry. Trying to develop innovative modes of transport becomes an inevitable trend. Hydraulic Capsule Pipelines (HCPs) are the freight transportation modes that use a kind of fluid to push capsules filled with bulk solids materials through water-filled pipelines. HCPs not only alleviate everincreasing costs caused by energy scarcities and oil price up, but also solve issues like traffic congestion and environmental pollution. Published literature is mainly limited to numerical simulation of the unidirectional fluid-structure interaction between the capsules and the fluid inside the pipelines; furthermore, the hydraulic characteristics only involve the speed of the capsules and the pressure drop characteristics of the fluid within the pipe. This research was conducted on the following four aspects of HCPs. First, an improved cylindrical capsule called a "piped carriage" was evaluated. Second, an associated solution between the fluid domain within the pipe and the solid domain of the piped carriage was investigated numerically on the basis of the bidirectional fluid-structure interaction methods. Third, the effects of diameter ratio $b$ (ratio of a diameter of the piped carriage $D_{e}$ to a pipe diameter $D_{p}$, widely ranging in $b=0.4 \sim 0.95$ ) on hydraulic characteristics of transporting the piped carriage within the pipeline were extensively discussed. Finally, based on Least-Cost Principle, an optimization model of HCPs was effectively built. The results showed that the simulated results were in good agreement with the experimental results, which further indicated that it was feasible for solving the hydraulic characteristics of transporting the piped carriage by using the bidirectional fluid-structure interaction methods. The results will be of great reference value for further research on HCPs and also provide a theoretical foundation for the optimal design of HCPs.

\section{Introduction}

Hydraulic Capsule Pipelines (HCPs) have been becoming very popular in global freight transportation industries. In 1958, the concept was first put forward at the Alberta Research Council in Canada, and then the National Science Foundation in the United States set up a Capsule Pipeline Research Center at the University of Missouri-Columbia to further explore key technologies of HCPs since the early 1990s [1]. The principle of HCPs was to use capsules to enclose and transport cargoes through a water-filled pipeline with the aid of water thrust force. HCPs had several advantages such as low transport cost, high transportation efficiency, and green environmental protection. The speed of the capsules that were designed to be hollow containers usually should not exceed $5 \mathrm{~m} \cdot \mathrm{s}^{-1}$, which was suitable for transporting low-speed bulk solid materials such as grains and other agricultural products, industrial building materials, and harmful household waste [2]. When compared with the slurry hydraulic pipeline, HCPs had several superiorities of not being contaminated by the carrier fluid for cargoes, not requiring mechanical equipment to separate the transported material from the fluid and not improving high power to maintain the system operating. 
In recent decades, several researches on HCPs have been conducted. The first notable investigative study started in 1962 when Charles [3] adopted a series of theoretical methods to analyze the flow characteristics within the pipeline transporting a cylindrical capsule with the density equal to that of its carrier fluid (water). Later, based on the frictional resistance coefficient of a cylindrical capsule, Newton et al. [4], Ellis [5], and Kruyer et al. [6] extended Charles's [3] research work by predicting the effects of four factors, namely, axial displacement of the capsule from concentric position, end configuration, length of the capsule, and deformed annular cross-sections on the speed coefficient of the capsule. They ignored the effects of end shape of the capsule on the flow characteristics. Since mid-1970s, researchers started a wide range of experimental investigations on energy losses within the pipes, carrying a train of capsules. Latto et al. [7] and Tomita et al. [8] discussed the effects of the speed coefficient and the pressure drop coefficient on the flow characteristics for the train of capsules. Fujiwara et al. [9], Lenau et al. [10], Yanaida et al. [11], and Agarwal et al. [12] supplemented Tomita et al's [8] research findings by solving the instantaneous energy losses of the cylindrical capsule train inside the pipelines based on the methods of characteristics. However, the capsule was assumed to be a point mass, and a limited discussion on the flow velocity and pressure distributions in the vicinity of the capsules has been reported. In the early 1980s, many researchers led extensive research projects on the study of vertical HCPs and $90^{\circ}$ bending HCPs transporting cylindrical capsules. Hwang et al. [13], Polderman [14], and Tachibana et al. [15] discussed energy losses of the flow of cylindrical capsules by the three loss factors such as viscidity resistance, inflow contraction, and outflow expansion in a vertical pipeline. A wide range of experimental investigations on the hydrodynamics characteristics of capsules conveyed by water in the $90^{\circ}$ horizontal bends and straight inclined pipes, respectively, were conducted by Mishra et al. [16] and Vlasak et al. [17].

In addition to a cylindrical capsule, the transport features of the single spherical capsule were evaluated for HCPs analysis. A series of test data was used by Ellis et al. [18] and Teke et al. [19] to identify the pressure drop characteristics of the two-phase flow of both spherical ice capsules and the water. Ulusarslan [20] extended Teke et al.s [19] studies by analyzing the pressure drop characteristics caused by a train of the spherical capsules. An extension of this work was made by Barthès-biesel [21], Ulusarslan [22], and Dupont et al. [23] to analyze the effects of the diameter ratio on both the speed coefficient and the pressure gradient for the spherical capsule train whose density was lower than that of water. Such studies were limited to a spherical capsule whose density was similar to that of ice capsules.

In the late 1980s, several researchers started emphasizing the study on the flow characteristics in the annular slit space. They attempted to further obtain the dynamic characteristics of capsules transport by analyzing the flow characteristics of the annular slit flow. Garner et al. [24], Kumar et al. [25], and Markatos et al. [26] studied the flow characteristics of both laminar and turbulent flows between a circular pipe and an eccentric moving cylindrical capsule. Garg
[27], Ogawa et al. [28], and Kroonenberg [29] introduced respective friction coefficients between the moving capsule wall and the pipe wall and established mathematical models of transporting a concentric cylindrical capsule within the pipelines. Flow characteristics of both the developing and the fully developed annular slit flows were estimated by Sud et al. [30] and Tomita et al. [31], through studying fundamental relationships between the turbulent flow and Von Karman's similarity hypothesis [24] in an idealized model of a highspeed ground transportation system. Their studies were restricted to bidimensional flow conditions. Since the mid1990s, researchers broadened the research range of the fluid density, added to new application areas of HCPs technologies. The mathematical models of both the lubricating fluid and the Bingham fluid flowing in the concentric annular slit space with an axially moving capsule were established by Polderman et al. [32] and Huang et al. [33].

Since the early 1990s, as computer technologies mature, numerical simulation was being widely applied to solve complex hydraulics problems. The numerical analysis in the area of HCPs has been reported by Khalil et al. [34] who simulated the flow of the cylindrical capsule within the pipelines. A comparison of the three different turbulent models was adopted for the analyses of both the velocity profiles and the pressure drops. Asim et al. [35, 36] and Wang et al. [37] used a validated computational fluid dynamics tool to numerically simulate the solid-liquid two-phase flow in both on-shore and off-shore HCPs and proposed a semiempirical model for predicting the pressure drops of HCPs. These studies focused only on the unidirectional fluid-structure interaction such that the capsule movement was assumed to be uniform motion with a constant speed within the pipelines. Feng et al. [38] conducted a series of analytical studies on a bidirectional fluid-structure interaction problem between elliptic capsules and laminar fluid and divided motion stages of the elliptic capsules with the density heavier than that of its carrying fluid in a hydraulic pipe. The numerical simulation of the bidirectional fluid-structure interaction was involved, but a very limited discussion on the elliptic capsules carried by a Poiseuille flow in a bidimensional channel was been presented, which did not reflect the bidirectional fluidstructure interaction between the capsules and a tridimensional turbulent fluid.

It was not until Sun et al. [39] improved the structure of the capsule within the pipelines in 2007 at the $20^{\text {th }}$ national conference on hydrodynamics and named it a "piped carriage". In recent years, many scholars studied the hydraulic characteristics of transporting the piped carriage within the pipelines. The flow of the piped carriage in HCPs was studied experimentally by Sun et al. [39] who analyzed the hydraulic characteristics of the flow field under the start-up condition of the piped carriage within the pipelines and compared the energy losses between the flow of piped carriage and the pipe fluid which would exist if the fluid alone flowed in the same pipe at the same average axial velocity. Li et al. [40] and Wang et al. [41] extended Sun et al.s [39] work by using model experiments to develop a semiempirical formula between the speed of the piped carriage and the average axial velocity of the pipe fluid. Combined with the 
Navier-Storkes equations, Zhang et al. [42], Li et al. [43], and Zhang et al. [44] built several mathematical models on concentric annular slit flow around a combinational cylindrical body with a special array of cylinders under moving and static boundary conditions and analyzed the flow characteristics of the concentric annular slit flow. The hydraulic characteristics of the flow field behind the piped carriage within the horizontal pipeline under different Reynolds numbers was presented by Jing et al. [45] and Guo [46]. Due to restrictions with testing equipment, their studies were not able to fully obtain the complicated hydraulic characteristics of transporting the piped carriage through the pipelines.

In recent years, the numerical simulation of a bidirectional fluid-structure interaction has become an important tool of fluid machinery analysis. In the bidirectional fluidstructure interaction methods, interaction between the fluid within the pipeline and the structural responses of the solid was considered and the simultaneous solution of fluid domains and solid domains was involved, which made computational results much more close to real physical phenomena. At present, the numerical simulation of the bidirectional fluid-structure interaction has mainly obtained some research findings in the rotating machineries such as the centrifugal water pumps, the fans, and the turbines $[47,48]$, but studies on the bidirectional fluid-structure interaction of transporting the piped carriages in HCPs were limited. There was a significant bidirectional fluid-structure interaction between the pipe fluid and the piped carriage. That is, on the one hand, the piped carriage yielded dynamic responses due to the action of the fluid, but on the other hand, the dynamic responses of the piped carriage in turn affected flow characteristics of the flow field. If the piped carriage was generally considered as uniform motion with the same speed, the effect of the fluid on the dynamic responses of the piped carriage was neglected, which had the larger errors when compared with the experimental results. Therefore, to find a moving piped carriage through the water-filled pipeline, adopting the bidirectional fluidstructure interaction methods was indispensable.

On the basis of the previous published literature, the bidirectional fluid-structure interaction between the fluid domain within the pipelines and the solid domain of the piped carriage was numerically investigated by using the commercial CFD software ANSYS-Fluent 12.0. This approach improved the solution accuracy for both the flow field and the transient responses of the piped carriage. The effects of diameter ratio $b$ (ratio of a diameter of the piped carriage $D_{e}$ to a pipe diameter $D_{p}$ ) on the hydraulic characteristics also were evaluated by identifying velocity distributions, pressure distributions, vorticity magnitude distributions, speed coefficients, pressure drop characteristics, and mechanical efficiencies as well as force statistics during the movement of the piped carriage. At the same time, based on Least-Cost Principle, an optimization model of HCPs transporting the piped carriage was built effectively. This paper not only makes up for the shortcomings of the experimental investigations but also improves the computing accuracy of both the fluid domain within the pipelines and the solid domain of the piped carriage, providing a solid theoretical foundation for the rational determination of the technical elements in HCPs and an accurate evaluation of its transport effects.

The remainder of this paper is organized as follows. Section 2 expresses the theoretical analysis of the piped carriage. The solution method of the bidirectional fluidstructure interaction, including geometric model and governing equations of fluid domain as well as motion equations of solid domain, is analyzed in Section 3. Section 4 presents the materials and methods of the model experiments. In Section 5, the verification of the simulated results is shown to improve its reliability. Section 6 discusses the threedimensional velocity distribution, vorticity magnitude distribution, pressure distribution, velocity coefficient, mechanical efficiencies, force statistics, and pressure drop characteristics of moving piped carriages having different diameter ratios $b$ within the pipelines. In Section 7, an optimization model of HCPs is established based on Least-Cost Principle. The main conclusions from the research are summarized in Section 8.

\section{Theoretical Analysis}

2.1. Design of Piped Carriage. In recent years, cylindrical capsules have been widely studied throughout the world. Asim et al. [49] analyzed a cylindrical capsule with density equip to that of its carrier water, and this capsule collided violently with its conveying pipes when moving in an $90^{\circ}$ HCPs bend of bend-to-pipe radius ratios of 4 and 8. Agarwal et al. [50] studied and provided a cylindrical capsule with density heavier than that of its carrying fluid. When moving along the pipelines, this cylindrical capsule sank to the bottom of the pipe and rubbed against the inner wall of the pipe, which reduced the service life of the conveying pipe to some extent. Based on the above shortcomings of the cylindrical capsules, many experts and scholars have made a series of improvements on the cylindrical capsule. Later, the cylindrical capsule with wheels was proposed by Kosugi [51] that can reduce the frictional resistance of the cylindrical capsule transport within the pipelines. However, the cylindrical capsule with wheels only achieved a two-dimensional translation, and had the lower stability when moving within the pipelines. Ulusarslan et al. [52] extended their work by taking into account a flow of a spherical capsule, which had satisfied kinetic stability compared with the cylindrical capsule. However, because of the pipe diameter limitations, the interior volume of the spherical capsule was so small that it was not conducive to transporting a large amount of bulk solid materials. In the study, an improved cylindrical capsule called a "piped carriage" was presented, which effectively overcame many deficiencies of the cylindrical capsule, the spherical capsule, and the cylindrical capsule with wheels. Compared with more capsules in the previous published literature, the piped carriage had the advantages of obvious structural innovation. The piped carriage was comprised of a barrel, support bodies, and universal balls [39]. The physical diagram of the piped carriage was shown in Figure 1.

A barrel was an airtight hollow cylinder that machined from Plexiglas with a thickness of $5 \mathrm{~mm}$, and the front and rear end of the barrel were connected to screw caps by threads. The six support bodies were symmetrically installed 

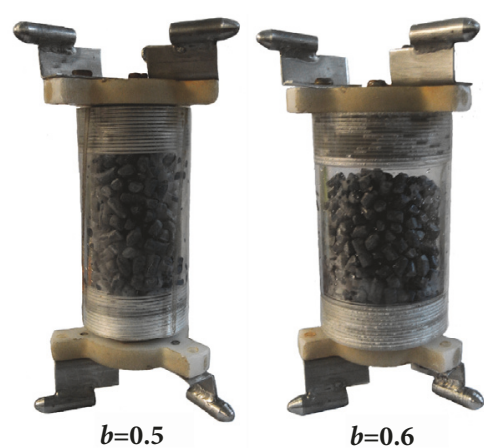

$b=0.6$
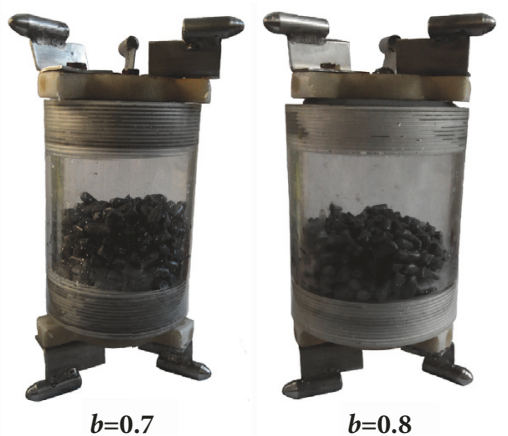

FIGURE 1: Physical diagram of a piped carriage.

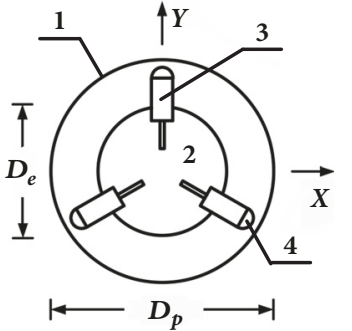

(a) Front view

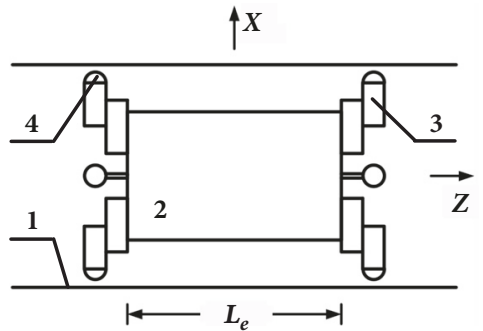

(b) Side view

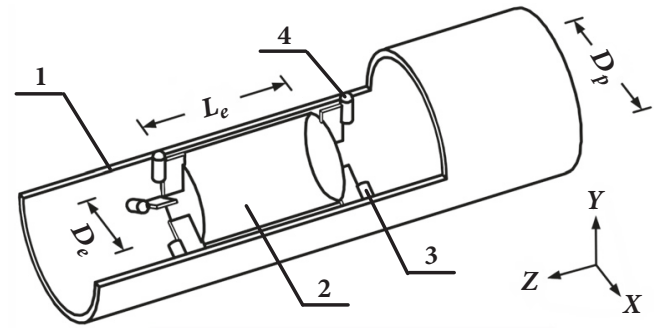

(c) Tridimensional model

FIGURE 2: Structure diagram of a piped carriage. Note: 1: pipeline. 2: barrel. 3: support bodies. 4: universal balls.

on the front and rear of the piped carriage at 120 degree angles from each other. The support bodies maintained the concentric position of the piped carriage within the horizontal pipeline, which can prevent the piped carriage from severe vibrations caused by the velocity variation that occurred suddenly throughout the pressure pipelines. The support bodies thus improved the stability of the piped carriage during transport. The support bodies did not lead to the tilt of transporting the piped carriage and avoided the collision between the piped carriage and the conveying pipe. The universal balls were mounted at the end of the support bodies to reduce the frictional resistance between the outer wall of the piped carriage and the inner wall of the pipes [40], thus contributing to energy efficiency. In addition, the universal balls can make the piped carriage translational and rotated. As discovered by research, there was no collision between the piped carriage and the conveying pipes, only the frictional resistance coefficient needed to be considered. Steel ball bearings having a diameter of $8 \mathrm{~mm}$ were served inside the piped carriage and defined as a basic transport loading of the piped carriage. The space between steel ball bearings was filled with rubber marine sand which possessed gelling property so that the stability of the piped carriage was improved effectively. The carriers in the previous published literature did not consider the transport loading. In this study, the transport loading was first put forward in HCPs, which will provide a theoretical basis for engineering application of the piped carriage. The structure diagram of the piped carriage was shown in Figure 2.

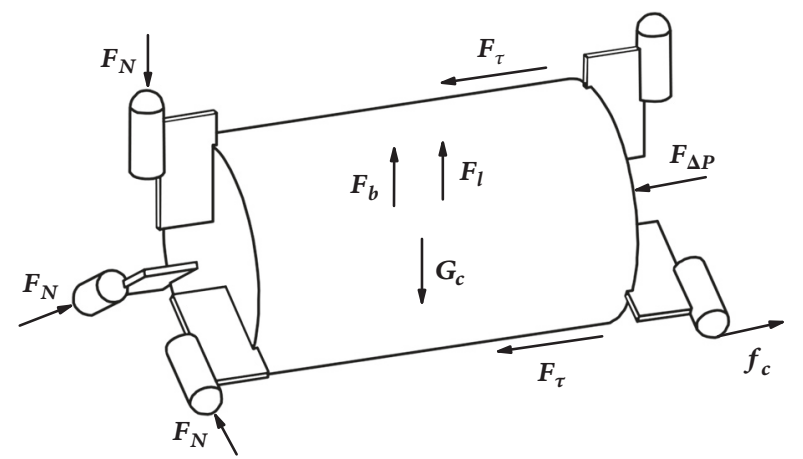

FIGURE 3: The force analysis on the piped carriage.

2.2. Force Analysis. There were obvious differences in the structure between the piped carriage and the other carriers mentioned in the previous published literature, such as cylindrical capsule, spherical capsule, and cylindrical capsule with wheels. From the perspective of fluid dynamics, forces acting on a piped carriage transport had its own characteristics. Taking the piped carriage as a main research object, the forces acting on the piped carriage in the horizontal pipeline were analyzed. According to the direction of pipe axis, the forces acting on the piped carriage can be divided into two categories. The force analysis on the piped carriage was shown in Figure 3. 
2.2.1. Forces in the Direction of the Pipe Axis. (1) The pressure gradient force acting on the front and rear ends of the piped carriage, $F_{\triangle p}$, which was written as follows:

$$
F_{\Delta P}=\Delta p A_{e}=F_{p 1}-F_{p 2}
$$

where $\triangle p$ was the pressure difference between the pipe fluid acting on the front end and rear end of the piped carriage; $A_{e}$ was the end-face areas of the piped carriage; $F_{p 1}$ and $F_{p 2}$ were the fluid pressure acting on the rear and front ends of the piped carriage, respectively.

(2) The shear stress of annular slit flow acting on the sidewall of the piped carriage, $\tau_{e}$. If the average axial velocity of the annular slit flow was greater than the average speed of the piped carriage, the shear stress was equivalent to power; otherwise, it would be equivalent to the resistance. It was written as follows:

$$
\tau_{e}=0.125 \rho \lambda_{e}\left(V_{s}-V_{b}\right)^{2}
$$

where $\lambda_{c}$ was the flow resistance coefficient; $\rho$ was the density of the fluid; $V_{s}$ was the average axial velocity of the annular slit flow; $V_{b}$ was the average speed of the piped carriage.

(3) The rolling frictional resistance acting on the piped carriage, $f_{c}$. There was no rolling frictional resistance when the cylindrical capsule and the spherical capsule moved along the pipelines. The rolling frictional resistance can be written as follows:

$$
f_{c}=F_{N} \mu_{c}
$$

where $F_{N}$ was the support force of the pipe wall against the piped carriage; $\mu_{c}$ was the rolling frictional resistance coefficient.

$$
F_{d}=F_{\Delta P}+\pi \tau_{e} D_{e} L_{e}
$$

where $F_{d}$ was the drag of the piped carriage.

2.2.2. The Forces Perpendicular to the Pipe Axis. (1) The gravity of the piped carriage, $G_{c}$. It was composed of the weight of both the empty piped carriage itself and the transport loading.

(2) The support force of the pipe wall against the piped carriage, $F_{N}$. The support forces in different directions acted on the contact points between the universals of the piped carriage and the inner wall of pipe, and both pointed to the center of the conveying pipe.

(3) The buoyancy of the piped carriage, $F_{b}$. It was written as follows:

$$
F_{b}=\rho g C_{c}
$$

where $C_{c}$ was the interior volume of the piped carriage.

(4) The lift of the piped carriage, $F_{l}$, which was related to fluid pressure and flow velocity.

2.3. Motion Stages. The motion processes of the piped carriage through a horizontal pipeline can be divided into three basic stages.
2.3.1. Static Stage. Since resultant force between pressure gradient force and shear stress can be inadequate to restrain the maximum static rolling frictional resistance acting on the piped carriage, the piped carriage was in static stage. The static stage was written as follows:

$$
F_{\Delta P}+\pi \tau_{e} D_{e} L_{e}<f_{\text {cmax }}
$$

where $f_{\text {cmax }}$ was the maximum static rolling frictional resistance.

2.3.2. Accelerative Stage. When pipe discharge within the pipelines increased, resultant force between pressure gradient force and shear stress was greater than rolling frictional resistance; thereby the piped carriage entered into accelerative stage. The accelerative stage was written as follows:

$$
F_{\Delta P}+\pi \tau_{e} D_{e} L_{e}>f_{c}
$$

When pipe discharge was constant, resultant force between pressure gradient force and shear stress acting on the piped carriage was gradually decreased with the increasing of instantaneous speed of the piped carriage. Therefore, accelerated speed of the piped carriage was gradually decreased in the accelerative stage.

2.3.3. Steady Stage. When instantaneous speed of the piped carriage reached the maximum value, the piped carriage entered into steady stage. At the same time, the piped carriage realized a force balance in the direction of the pipe axis. The steady stage was written as follows:

$$
F_{\Delta P}+\pi \tau_{e} D_{e} L_{e}=f_{c}
$$

Due to a short period of the accelerative stage of the piped carriage, the steady stage of the piped carriage transport was mainly studied in this paper. Tomita et al. [31], Khalil et al. [34], and Asim et al. [36] considered the steady stage of a cylindrical capsule transport. However, the cylindrical capsule did not have rolling frictional resistance. In fact, the steady stage of the piped carriage tended to a dynamical equilibrium; the reason was that the pulsation of the pipe fluid caused the instantaneous speeds of the piped carriage to fluctuate within a certain range.

As described by Liu et al. [53], motion processes of a cylindrical capsule can be classified into four stages, and the speed of the cylindrical carriage at each stage had specific requirements. In the fourth steady stage, for the cylindrical capsule, the carrier speed was greater than the average axial velocity of the pipe fluid. But for the piped carriage, the carrier speed had no direct relation to the average axial velocity of the pipe fluid. Through the above comparison, it can be obtained that speeds between the cylindrical carriage and the piped carriage were of essential difference in the steady stage.

2.4. Motion Model. According to the force characteristics, the motion model of the piped carriage in the steady stage was established. Using the transported piped carriage as a reference frame, the flow conservation equation was written as follows:

$$
\rho \pi D_{p}^{2}\left(V_{a}-V_{b}\right) \Delta t=\rho \pi\left(D_{p}^{2}-D_{e}^{2}\right)\left(V_{s}-V_{b}\right) \Delta t
$$


where $V_{a}$ was the average axial velocity of the pipe fluid; $\Delta t$ was the motion period.

The relationship among the average axial velocity of the pipe fluid $\left(V_{a}\right)$, the pipe discharge, and mixture pipe discharge was defined. The pipe discharge in this manuscript was the fluid mass per hour for the single-phase flow at the inlet cross-section of the conveying pipe, and the mixture pipe discharge was the fluid mass per hour for the solid-liquid two-phase flow within the pipeline. The pipe discharge and mixture pipe discharge are different in physical explanations, but the same in magnitude. The average axial velocity of the pipe fluid $\left(V_{a}\right)$ was the ratio of the pipe discharge or the mixture pipe discharge to the pipe cross-sectional areas.

Let $b=D_{e} / D_{p}$

The average axial velocity of the annular slit flow was written as follows:

$$
V_{s}=\frac{\left(V_{a}-b^{2} V_{b}\right)}{\left(1-b^{2}\right)}
$$

When the piped carriage transported steadily along the pipe axis within the pipeline, the forces acting on the piped carriage and the annular slit flow in the part where the piped carriage in the direction of the pipe axis should both reached equilibrium states. When the average axial velocity of the annular slit flow was greater than the average speed of the piped carriage, the direction of the shear stress acting on the piped carriage was the same as the direction of the pipe fluid, then the shear stress was the power of the piped carriage; otherwise the shear stress was the resistance of the piped carriage.

If the shear stress acting on the sidewall of the piped carriage was power, the motion model of the piped carriage was deduced in this paper.

For the piped carriage, the force balanced equation along the pipe axis was written as follows:

$$
\Delta p \pi D_{e}^{2}+4 \tau \pi D_{e} L_{e}=4 f_{c}
$$

For the annular slit flow in the part where the piped carriage, the force balanced equation along the pipe axis was written as follows:

$$
\Delta p \pi\left(D_{p}^{2}-D_{e}^{2}\right)=4 \tau_{e} \pi D_{e} L_{e}+4 \tau_{p} \pi D_{p} L_{e}
$$

The shear stress acting on the interior wall of the conveying pipe was written as follows:

$$
\tau_{p}=0.125 \rho \lambda_{p} V_{s}^{2}
$$

where $\lambda_{p}$ was the friction coefficient of the pipe wall.

Equations (2), (3), and (10) substituted in (11) to obtain

$$
\Delta p=\frac{4 F_{N} \mu_{c}}{\pi b^{2} D_{p}^{2}}-\frac{\lambda_{e} \rho\left(V_{a}-V_{b}\right)^{2} L_{e}}{2 b D_{p}\left(1-b^{2}\right)^{2}}
$$

Equations (3), (10), and (13) substituted in (12) to obtain

$$
\begin{aligned}
\Delta p= & \frac{b \lambda_{e} \rho\left(V_{a}-V_{b}\right)^{2} D_{p} L_{e}}{2\left(1-b^{2}\right)^{3} D_{p}^{2}} \\
& -\frac{\lambda_{p} \rho\left(V_{a}-b^{2} V_{b}\right)^{2} D_{p} L_{e}}{2\left(1-b^{2}\right)^{3} D_{p}^{2}}
\end{aligned}
$$

Therefore, the motion model of the piped carriage was deduced from (14) and (15) and it can be written as follows:

$$
A_{1}\left(\frac{V_{b}}{V_{a}}\right)^{2}+A_{2}\left(\frac{V_{b}}{V_{a}}\right)+A_{3}=0
$$

where

$$
\begin{aligned}
A_{1}= & \left(\lambda_{e}+b^{3} \lambda_{p}\right) \frac{b \rho L_{e}}{2\left(1-b^{2}\right)^{3} D_{p}}+\frac{\rho \lambda_{e} L_{e}}{2 b\left(1-b^{2}\right)^{2} D_{p}}, \\
A_{2}= & -\left(\lambda_{e}+b \lambda_{p}\right) \frac{b \rho L_{e}}{\left(1-b^{2}\right)^{3} D_{p}}-\frac{\rho \lambda_{e} L_{e}}{b\left(1-b^{2}\right)^{2} D_{p}}, \\
A_{3}= & \left(b \lambda_{e}+\lambda_{p}\right) \frac{\rho L_{e}}{2\left(1-b^{2}\right)^{3} D_{p}}+\frac{\rho \lambda_{e} L_{e}}{2 b\left(1-b^{2}\right)^{2} D_{p}} \\
& -\frac{4 F_{N} \mu_{c}}{\pi b^{2} D_{p}^{2} V_{a}^{2}}
\end{aligned}
$$

Equation (16) was only applicable to the case, where the shear force acting on the sidewall of the piped carriage was power.

If the shear stress acting on the sidewall of the piped carriage was resistance, the motion model of the piped carriage was written as follows:

$$
A_{4}\left(\frac{V_{b}}{V_{a}}\right)^{2}+A_{5}\left(\frac{V_{b}}{V_{a}}\right)+A_{6}=0
$$

where

$$
\begin{aligned}
A_{4}= & \left(-\lambda_{e}+b^{3} \lambda_{p}\right) \frac{b \rho L_{e}}{2\left(1-b^{2}\right)^{3} D_{p}} \\
& -\frac{\rho \lambda_{e} L_{e}}{2 b\left(1-b^{2}\right)^{2} D_{p}}, \\
A_{5}= & \left(\lambda_{e}-b \lambda_{p}\right) \frac{b \rho L_{e}}{\left(1-b^{2}\right)^{3} D_{p}}+\frac{\rho \lambda_{e} L_{e}}{b\left(1-b^{2}\right)^{2} D_{p}}, \\
A_{6}= & \left(-b \lambda_{e}+\lambda_{p}\right) \frac{\rho L_{e}}{2\left(1-b^{2}\right)^{3} D_{p}}-\frac{\rho \lambda_{e} L_{e}}{2 b\left(1-b^{2}\right)^{2} D_{p}} \\
& -\frac{4 F_{N} \mu_{c}}{\pi b^{2} D_{p}^{2} V_{a}^{2}}
\end{aligned}
$$

Equations (16) and (18) were the motion models of the piped carriage obtained by using the force analysis. Because the parameters such as $\lambda_{e}$ and $\lambda_{p}$ in this motion model cannot 


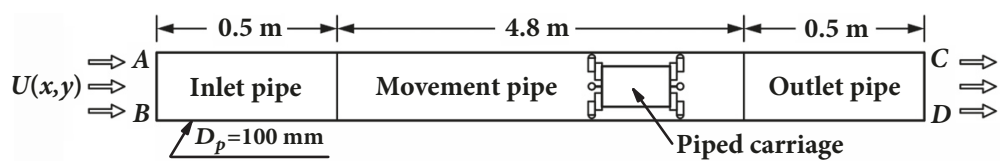

FIGURE 4: Geometric model. Note: $U(x, y)$ represents distribution function of axial velocity at inlet cross-section. $A B$ represents inlet crosssection. $C D$ represents outlet cross-section.

be obtained accurately, it can only be used to qualitatively analyze the relationship between the average speed of the piped capsule and other impact factors such as pipe fluid, transport loading, and structure of the piped carriage. The motion model was of great significance to the analysis of the average speed coefficient of piped carriage in Section 6.1.

\section{Fluid-Structure Interaction Method}

3.1. Geometric Model. A geometrical model of a piped carriage transported in a horizontal pipe was created using the preprocessing software Pro/E wildfire 4.0. In order to improve the quality of meshes, the geometric model was simplified appropriately during the modeling processes. In this paper the original model was used for the numerical simulation, which can greatly improve the calculation accuracy of the simulated results. The geometry model included a horizontal pipe model and a piped carriage model, as shown in Figure 4.

The horizontal pipe model was $5.8 \mathrm{~m}$ long with a diameter of $100 \mathrm{~mm}$, exactly the same as the size of test section on the experimental system. To improve the precision of simulation, the horizontal pipe model was divided into three sections: an inlet pipe, a movement pipe and an outlet pipe, where the lengths of these three sections were $0.5 \mathrm{~m}, 4.8 \mathrm{~m}$, and 0.5 $\mathrm{m}$, respectively. A $0.5 \mathrm{~m}$ long inlet pipe was used in order to allow the pipe fluid to become fully developed. A $0.5 \mathrm{~m}$ long outlet pipe was used in order to reduce the effects of the piped carriage on the pressure of the outlet cross-section in the geometric model. A $4.8 \mathrm{~m}$ long movement pipe was the range of motion for the center of the piped carriage. The movement pipe with an inner diameter of $0.1 \mathrm{~m}$ was similar to that of Ulusarslan [22] and Asim et al. [35]. The diameters of the piped carriage model varied from 40 to $95 \mathrm{~mm}$ with an interval of $5 \mathrm{~mm}$, and the length of the piped carriage was 0.1 $\mathrm{m}$. The support of the piped carriage was composed of a thin cylinder and a sheet-metal plate. The thin cylinder was $0.02 \mathrm{~m}$ in length and $8 \mathrm{~mm}$ in diameter. A $0.01 \mathrm{~m} \times 0.015 \mathrm{~m} \times 0.001 \mathrm{~m}$ sheet-metal plate played a significant role in connecting the barrel and the thin cylinder. The universal ball mounted at the end of the support body was a hemispherical structure with a spherical radius of $4 \mathrm{~mm}$. In the study, the piped carriage model always maintained a concentric position with the horizontal pipe model. In the initial state, the center of the piped carriage model was $0.7 \mathrm{~m}$ away from the inlet cross-section of the geometric model. The initial location of the piped carriage was shown in Figures 2(a) and 2(b), in which $Z$ was defined as the direction of the water, and $X$ and $Y$ were defined as the direction of horizontally left and the direction of vertically upward, respectively. The pipe discharges of the geometrical model were $40,50,60$, and 70 $\mathrm{m}^{3} \cdot \mathrm{h}^{-1}$, respectively.

3.2. Governing Equations of Fluid Domain. The meshing of the geometric model was completed using the software ICEM CFD. The computational domains of this paper were divided into two parts: a fluid domain and a solid domain. The fluid domain of the geometric model included the inlet pipe, the movement pipe, and the outlet pipe of three parts. A hybrid meshes of the same size were used for the meshing of the fluid domain. It meant that the two different mesh types have been created in the fluid domain. The inlet and outlet pipes were meshed with hexahedral elements. The reason for this was that the hexahedral mesh elements can be generated in these pipes with a very low skewness and provided more accurate results due to lower numerical diffusion. The movement pipe was meshed with tetrahedral elements, which can improve the quality of meshes near the exterior wall of the piped carriage. In order to reduce the effects of mesh size on the simulated results, a mesh independence test was performed for the fluid domain of the geometric model [54]. Through comparing the effects of different mesh sizes on the average pressure of inlet cross-section in the geometrical model under the pipe discharge of $50 \mathrm{~m}^{3} \cdot \mathrm{h}^{-1}$, a reasonable mesh size was obtained successfully, as shown in Table 1.

The average pressure at the inlet section-section of the geometric model had the following advantages as a main index of the mesh independence test: (1) Because pressure distributions at the outlet cross-section of the geometric model was the same, the pressure at the inlet cross-section of the geometric model can indirectly analyze the pressure drop of the pipe fluid. (2) The index can be measured by the model experiments to further verify the correctness of the simulated results at the inlet cross-section of the geometric model.

According to simulated results of the mesh independence test in Table 1, the relative errors of the average pressure of the inlet cross-section in the geometrical model under the six different diameter ratios were not more than $0.43 \%$ between mesh sizes of both $0.002 \mathrm{~m}$ and $0.003 \mathrm{~m}$. When the mesh size was less than $0.002 \mathrm{~m}$, the effects of the mesh size on the average pressure of inlet cross-section were negligible. At last, through the comparison between simulated results and experimental results, both results were in qualitatively good agreement. Based on a comprehensive analysis, when the mesh size of the fluid domain was 0.002 $\mathrm{m}$, the simulated results have reached the requirement of the mesh independence test. In the study, to efficiently attain the desired solution accuracy, local surface meshes were used to achieve secondary mesh refinement for the solid domain of the piped carriage, in which the meshes consisted of 
TABLE 1: Mesh independence test.

\begin{tabular}{|c|c|c|c|c|c|c|}
\hline \multirow{2}{*}{$\begin{array}{l}\text { Mesh } \\
\text { size/m }\end{array}$} & \multicolumn{6}{|c|}{ Average pressure of inlet cross-section/Pa } \\
\hline & $b=0.40$ & $b=0.50$ & $b=0.60$ & $b=0.70$ & $b=0.80$ & $b=0.90$ \\
\hline 0.006 & 12495.39 & 12461.09 & 12408.27 & 12386.75 & 12512.26 & 13230.65 \\
\hline 0.005 & 12353.32 & 12308.47 & 12259.92 & 12243.51 & 12365.11 & 12938.23 \\
\hline 0.004 & 12240.71 & 12204.73 & 12142.14 & 12119.87 & 12249.96 & 12805.02 \\
\hline 0.003 & 12166.49 & 12121.09 & 12073.33 & 12046.39 & 12172.06 & 12707.18 \\
\hline 0.002 & 12116.31 & 12078.85 & 12040.78 & 11994.49 & 12130.52 & 12657.67 \\
\hline
\end{tabular}

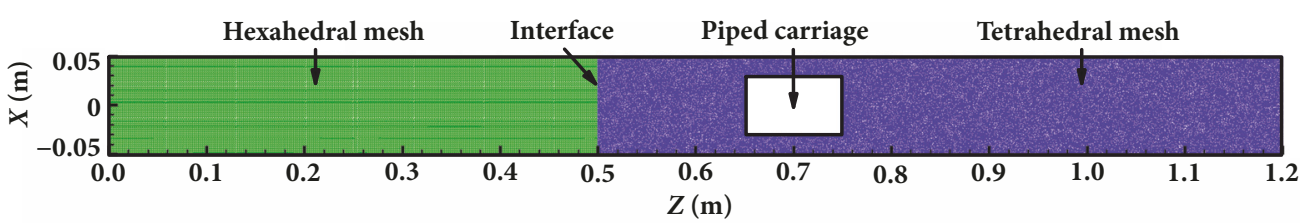

(a) Meshing of the fluid domain within a pipeline

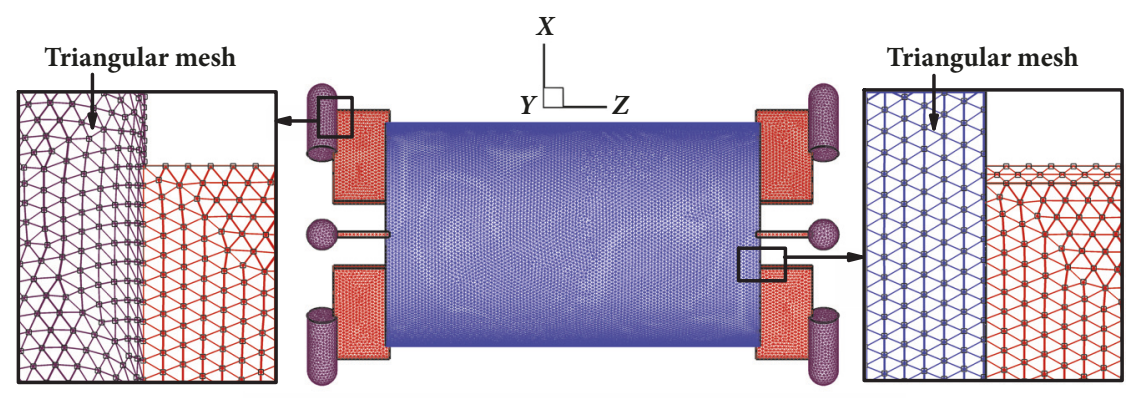

(b) Meshing of the solid domain for a piped carriage

FIGURE 5: Meshing of computational domains in geometric model.

triangular elements with a specific size of $0.001 \mathrm{~m}$ to reduce its skewness. Boundary layer areas were also meshed with rectangle elements. According to the calculation formulas of the boundary layer areas [55], the boundary layer thickness of the pipe wall in the turbulent areas was determined. The thickness of first layer mesh was to be $0.2537 \mathrm{~mm}\left(y^{+}=30\right)$ by calculation. It is noteworthy that the $y^{+}$values were such that the first mesh node is in the log-law region. The scale factor between mesh layers was 1.2, and the mesh number in this areas was set to 11. At the initial time, the meshing of computational domains in geometric model (the diameter ratio of the piped carriage was 0.6) was shown in Figure 5.

Taking into account the complexity of the flow field caused by transport of a piped carriage, the following assumptions were used in the simulation algorithm: (1) The fluid density was considered to be constant, thus ignoring any change of density. (2) The energy equation was ignored due to nonexisting heat exchange between the fluid and the pipe wall.

The fluid medium was defined as liquid-water with a density of $1000 \mathrm{~kg} \cdot \mathrm{m}^{-3}$ and dynamic viscosity of $1.062 \times 10^{-3} \mathrm{~Pa} \cdot \mathrm{s}$ (the water temperature was $18^{\circ} \mathrm{C}$ ). The operating conditions being given to the solver were the operating pressure of 101
$325 \mathrm{~Pa}$ (i.e., atmospheric pressure) and ignored the effects of the gravity on the flow field within the pipeline. Investigations had been carried out on the support bodies made of stainless steel having a density of $7.93 \times 10^{3} \mathrm{~kg} \cdot \mathrm{m}^{-3}$ and the barrel made of Plexiglas having a density of $1.2 \times 10^{3} \mathrm{~kg} \cdot \mathrm{m}^{-3}$. The criterion for internal flows to be turbulent was that Reynolds number should be above 4000 . As it can be clearly seen that the Reynolds number under consideration was $>>4000$, it can be safely concluded that the flow inside the pipeline under discussion was turbulent. Hence, turbulent model needed to be introduced for numerical calculation. Compared with the standard $k-\varepsilon$ and the realizable $k-\varepsilon$ turbulent models, the RNG $k-\varepsilon$ turbulent model was far superior to other models in addressing flow problems having high strain rates and sharply curved streamlines. In the Cartesian coordinates, the time-continuous equation and the Reynolds equation for the incompressible viscous fluid in the form of a tensor index [56] were written as follows:

Continuity equation:

$$
\frac{\partial \rho}{\partial t}+\frac{\partial}{\partial x_{i}}\left(\rho u_{i}\right)=0
$$


Momentum equation (Navier-Stokes equation):

$$
\begin{aligned}
& \frac{\partial\left(\rho u_{i}\right)}{\partial t}+\frac{\partial}{\partial x_{j}}\left(\rho u_{i} u_{j}\right) \\
& =-\frac{\partial p}{\partial x_{i}}+\frac{\partial}{\partial x_{j}}\left[\mu \frac{\partial u_{i}}{\partial x_{j}}-\rho \overline{u_{i}^{\prime} u_{j}^{\prime}}\right]+S_{i}
\end{aligned}
$$

The turbulent model was composed of the turbulent kinetic energy equation and the turbulent dissipation rate equation.

Turbulent kinetic energy equation:

$$
\begin{aligned}
\frac{\partial(\rho k)}{\partial t}+\frac{\partial\left(\rho k u_{j}\right)}{\partial x_{j}}= & \frac{\partial}{\partial x_{j}}\left(\alpha_{k} \mu_{e f f} \frac{\partial k}{\partial x_{i}}\right) \\
& +\rho \overline{u_{i}^{\prime} u_{j}^{\prime}} \frac{\partial u_{i}}{\partial x_{j}}-\rho \varepsilon
\end{aligned}
$$

Turbulent dissipation rate equation:

$$
\begin{aligned}
& \frac{\partial(\rho \varepsilon)}{\partial t}+\frac{\partial\left(\rho \varepsilon u_{j}\right)}{\partial x_{j}} \\
& =\frac{\partial}{\partial x_{j}}\left(\alpha_{\varepsilon} \mu_{e f f} \frac{\partial \varepsilon}{\partial x_{i}}\right) \\
& \quad+\rho \overline{u_{i}^{\prime} u_{j}^{\prime}} \frac{\partial u_{i}}{\partial x_{j}} \frac{\varepsilon}{k}\left(C_{1 \varepsilon}-\frac{\eta\left(1-\eta / \eta_{0}\right)}{1+\beta \eta^{3}}\right) \\
& \quad-C_{2 \varepsilon} \frac{\rho \varepsilon^{2}}{k}
\end{aligned}
$$

where $t$ was the time; $\rho$ was the density of the fluid; $u_{i}$ and $u_{j}$ were the components of the time-averaged velocity components in the $i$ and $j$ directions, respectively; $u_{I}^{\prime}$ and $u_{j}^{\prime}$ were the fluctuating velocity in the $i$ and $j$ directions, respectively; $i$ and $j$ were 1,2 , and 3 , respectively. $p$ was the time-averaged pressure; $\mu$ was the dynamic viscosity; $x_{i}$ and $x_{j}$ were the coordinate components in the $i$ and $j$ directions, respectively; $S_{i}$ was the projection of the generalized source term of the momentum equation in the $i$ direction; $k$ was the turbulent kinetic energy; $\varepsilon$ was the turbulent dissipation rate; $\mu_{\text {eff }}=\mu+\mu_{t}$ was the effective viscosity; $\mu_{t}=\rho C_{\mu} k^{2} / \varepsilon$ was the turbulent viscosity, $C_{\mu}=0.0845, \mathrm{~Pa} \cdot \mathrm{s}$. $C_{1 \varepsilon}$ and $C_{2 \varepsilon}$ were the turbulent model coefficients, respectively, $C_{1 \varepsilon}=1.42, C_{2 \varepsilon}=1.68$. $\alpha_{k}$ and $\alpha_{\varepsilon}$ were the effective Prandlt numbers for $k$ and $\varepsilon$, respectively, $\alpha_{k}=\alpha_{\varepsilon}=1.39 .-\rho \overline{u_{i}^{\prime} u_{j}^{\prime}}=\mu_{t}\left(\partial u_{i} / \partial x_{j}+\partial u_{j} / \partial x_{i}\right)-$ $(2 / 3)\left(\rho k+\mu_{t}\left(\partial u_{i} / \partial x_{i}\right)\right) \delta_{i j}$ was the time-averaged stress tensor; $\eta_{0}$ and $\beta$ were constants, respectively, $\eta_{0}=4.377, \beta=0.012 . \eta=$ $\left(2 E_{i j} \cdot E_{i j}\right)^{1 / 2}(k / \varepsilon), E_{i j}=(1 / 2)\left(\partial u_{j} / \partial x_{i}+\partial u_{i} / \partial x_{j}\right) . \delta_{i j}$ was the Kronecker delta.

In the near-wall areas of the computational domain, the RNG k- $\varepsilon$ turbulent model was no longer applicable due to the lower Reynolds numbers. Therefore, the method of a standard wall function was adopted to solve for viscous flow at low Reynolds numbers in the near-wall area. Furthermore, it was assumed that the dimensionless fluid flow velocity could be described by logarithmic normal distributions outside the viscous sublayer areas.

Global flow parameters involved in the simulations and analysis included an average speed coefficient, $C_{v}$, average drag coefficient, $C_{d}$, average lift coefficient, $C_{l}$, and average pressure drop coefficient of HCPs, $C_{p}$; these were defined as follows [57]:

$$
\begin{aligned}
C_{v} & =\frac{V_{b}}{V_{a}}, \\
C_{d} & =\frac{2 F_{d}}{\rho\left(V_{a}-V_{b}\right)^{2} A_{v e}}, \\
C_{l} & =\frac{2 F_{l}}{\rho\left(V_{a}-V_{b}\right)^{2} A_{p a}}, \\
C_{p} & =\frac{\Delta P_{m}}{\Delta P_{n}}
\end{aligned}
$$

where $A_{v e}$ was the reference areas in the direction vertical to the flow; $A_{p a}$ was the reference areas in the direction parallel to the flow; $\Delta P_{m}$ was the total pressure drop of the flow of the piped carriage; $\Delta P_{n}$ was the total pressure drop that would exist if fluid alone flowed in the same pipe at the same average axial velocity.

The boundary conditions of the geometric model that have been specified as follows:

(1) The inlet boundary was set as a "Velocity-Inlet" boundary condition. Combined with the user defined functions (UDFs), the velocity distributions at the inlet crosssection was defined with the distribution function $U(x, y)$ of the measured velocity profiles, as shown in Figure 4. The integrated evaluated results of $\operatorname{Re}, I_{c}, k$, and $\varepsilon$ at the inlet boundary were calculated by using the semiempirical formulas [58].

In the study, these pipe fluid parameters, Reynolds number, Re, turbulence intensity, $I_{c}$, turbulent kinetic energy, $k$, turbulent dissipation rate, $\varepsilon$, were written as follows:

$$
\begin{aligned}
\operatorname{Re} & =\frac{V_{a} D_{p}}{v}, \\
I_{c} & =0.16 \mathrm{Re}^{-0.125}, \\
k & =1.5\left(V_{a} I_{c}\right)^{2}, \\
\varepsilon & =\frac{C_{\omega}^{0.75} k^{1.5}}{l}
\end{aligned}
$$

where $v$ was the kinematic viscosity; $l$ was the turbulence length scale; $C_{\omega}$ was the empirical constant of the turbulent model, generally taking 0.09 . The parameter values of the pipe fluid were shown in Table 2.

(2) The outlet boundary was set as a "Pressure-Outlet" boundary condition. The pressure at the outlet boundary was defined with the measured pressure.

(3) The boundary of the horizontal pipe model adopted the "Stationary-Wall" boundary condition [59]. The pipe has been considered to be hydrodynamically smooth, having a wall roughness constant of zero. 
TABLE 2: The parameter values of the pipe fluid.

\begin{tabular}{lcccc}
\hline Pipe discharge $/ \mathrm{m}^{3} \cdot \mathrm{h}^{-1}$ & 40 & 50 & 60 & 70 \\
\hline $\operatorname{Re}$ & 133211 & 166514 & 199817 & 233120 \\
$I_{c}$ & 0.0366059 & 0.0355990 & 0.0347969 & 0.0341328 \\
$\mathrm{k} / \mathrm{m}^{2} \cdot \mathrm{s}^{-2}$ & 0.0040228 & 0.0059446 & 0.0081788 & 0.0107114 \\
$\varepsilon / \mathrm{m}^{2} \cdot \mathrm{s}^{-3}$ & 0.0059893 & 0.0107589 & 0.0173627 & 0.0260228 \\
\hline
\end{tabular}

(4) The boundary conditions of the piped carriage needed to be defined using the six degrees of freedom coupling model of the moving mesh technology and UDFs. In addition to defining the dynamic characters such as moment of inertia and rolling friction resistance of the piped carriage, the UDFs also specified the migration scope and the initial state of the piped carriage. Only horizontal movement along the $Z$ direction and the rotary movement around the $z$-axis were considered, while movement in the other directions was strictly limited. The instantaneous speed of the piped carriage at the initial position was defined by the measured values of the instantaneous speed at which the center of the piped carriage passed through the corresponding cross-section.

(5) The connecting cross-section boundaries between the inlet pipe, the movement pipe, and the outlet pipe were set as "Interface" boundary condition, which realized data to exchange between the different mesh types of the flow fields.

To improve the solution precision of the transient flow field, the PISO velocity-pressure coupling algorithm within ANSYS-Fluent 12.0 was used, which could modify pressure twice for the flow field. The finite volume methods were used to discretize the governing equations of the fluid domain [60]. The convective terms of the momentum equation, the turbulent kinetic energy equation, and the turbulent dissipation rate equation were discretized with $2^{\text {nd }}$ order upwind scheme, while the diffusion terms of the above equations were discretized using $2^{\text {nd }}$ order central differential scheme since the backward difference yields to unstable solution. The convergence residuals of the discrete form were set to $1 \times 10^{-4}$, and the time step of the unsteady state was $1 \times 10^{-6} \mathrm{~s}$.

3.3. Motion Equations of Solid Domain. The six-freedom rigid body motion equations were followed to solve the speed, the displacement, and the attitude for the piped carriage at any time. The rigid body motion equations were written as follows [34, 61]:

$$
\begin{aligned}
F & =m a_{c}, \\
M & =I \alpha_{c}+\omega_{c} \times\left(I \omega_{c}\right),
\end{aligned}
$$

where $F$ was the acting forces on the piped carriage; $m$ was the mass; $a_{c}$ was the accelerated speed; $M$ was the moment; $I$ was the moment of inertia matrix; $\omega_{c}$ was the angular speed; $\alpha_{c}$ was the angular acceleration.

In ANSYS $^{-}$Fluent 12.0, the acting forces on the piped carriage in the six-freedom rigid body motion equations included fluid loadings and nonfluid loadings. The fluid loadings were the coupling forces that fluids exerted on the piped carriage, including the drag and the lift, and the nonfluid loadings were the rolling frictional resistance, the gravity, and the buoyancy of the piped carriage. The coupling forces on the piped carriage were calculated with the governing equations of the fluid domain, while the rolling frictional resistance of the piped carriage was defined with the UDFs. The rolling frictional resistance was determined as the product of the support force of the piped carriage multiplied by the rolling frictional resistance coefficient between the piped carriage wall and the Plexiglas pipe wall, which was written as follows:

$$
f_{c}=\left(F_{l}+F_{b}-G_{c}\right) \mu_{c}=F_{N} \mu_{c}
$$

Through the study in experiment, the rolling frictional resistance coefficient between the piped carriage and the Plexiglas pipe was obtained, $\mu_{c}=0.462$.

3.4. Fluid-Structure Interaction Methods. In the study, the numerical investigations (nonsteady state) using the RNG $k-\varepsilon$ turbulent model were conducted on the hydraulic characteristics of the piped carriage transported in the horizontal pipe. The six degrees of freedom coupling model and the UDFs were used to analyze the transient dynamics characteristics of the piped carriage. The coupling interfaces realized the real time exchange between the fluid domain information and the solid domain information. The coupled motion of the piped carriage gave rise to displacement effects of the rigid body. The geometric model meshes were modified in real time by exploiting the moving mesh technology of both elastic smoothing and local reconstruction to adapt to the transient displacement variation [62].

The flow diagram of the bidirectional fluid-structure interaction analysis was shown in Figure 6.

(1) The initial parameters: the speed, $V_{c}^{t}$, the angular speed, $\omega_{c}^{t}$, the angle, $\theta_{c}^{t}$, and the displacement, $L_{c}^{t}$, were set at the initial time $t$.

(2) The speed and the angular speed at time $t$ were regarded as the boundary conditions for the next iteration. Combined with the basic governing equations and the turbulent model, the hydraulic characteristics at time $t+\Delta t$ were solved to obtain the force $F^{t+\Delta t}$ and the moment $M^{t+\Delta t}$ for the piped carriage at time $t+\Delta t$.

(3) The speed and the displacement at time $t+\Delta t$ were calculated, which was written as follows:

$$
\begin{aligned}
& V_{c}^{t+\Delta t}=V_{c}^{t}+\Delta t\left(\frac{F^{t+\Delta t}}{m}\right), \\
& L_{c}^{t+\Delta t}=\frac{\left(V_{c}^{t}+V_{c}^{t+\Delta t}\right) \Delta t}{2}
\end{aligned}
$$




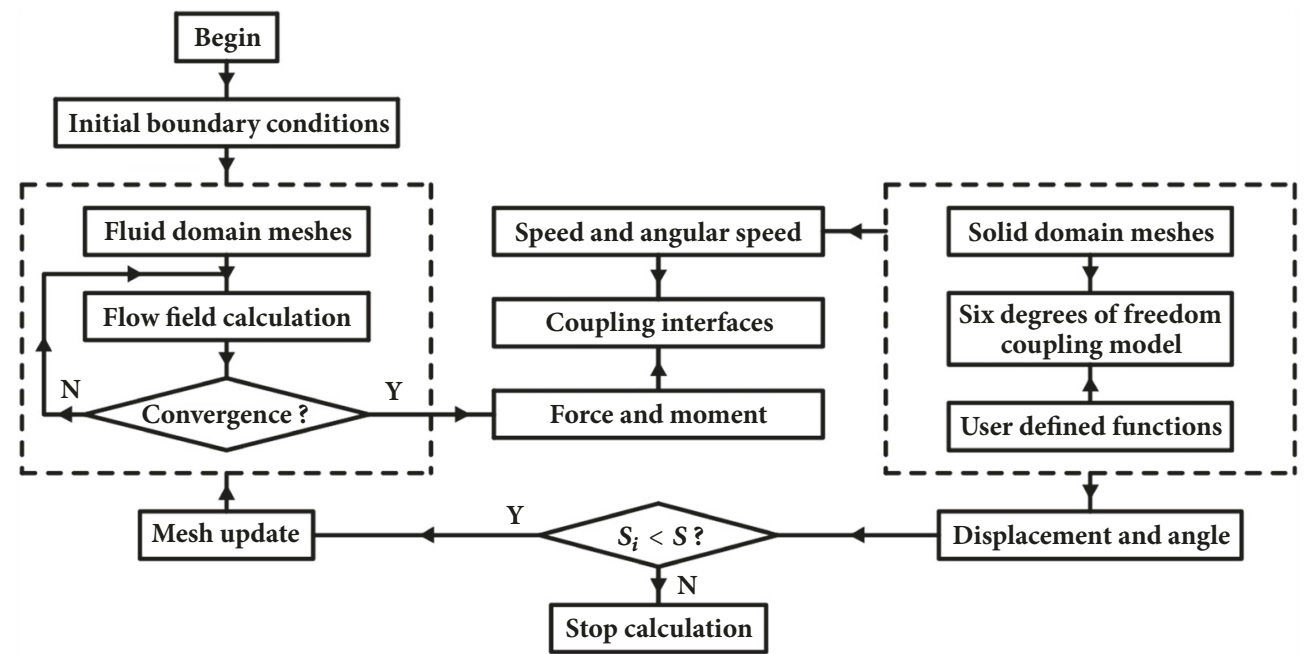

Figure 6: Flow diagram of bidirectional fluid-structure interaction analysis. Note: $S$ represents range of motion for piped carriage. $S_{i}$ represents displacement of piped carriage at any time.
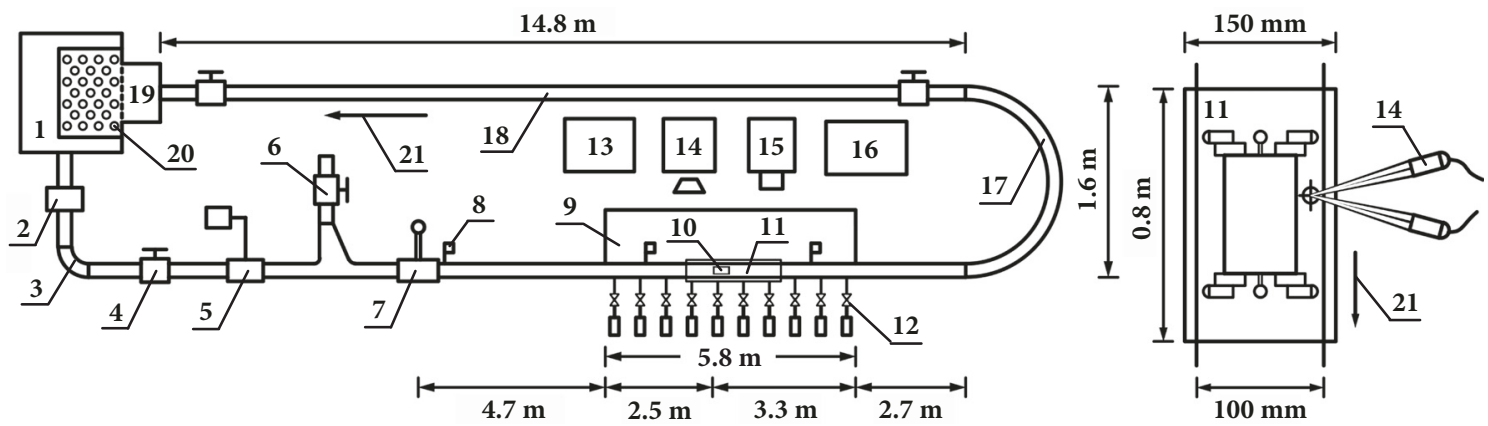

FIGURE 7: Experimental system. Note: 1: water tank. 2: steel penstocks. 3: centrifugal water pump. 4: regulating valve. 5: electromagnetic flowmeter. 6: input port for piped carriage. 7: braking device. 8: millisecond photoelectric timing devices. 9: test section. 10: piped carriage. 11: rectangular water channel. 12: pressure sensors. 13: standard dynamic pressure collection box. 14: Laser Doppler Anemometry. 15: high-speed camera. 16: personal computer. 17: $180^{\circ}$ pipe bend. 18: horizontal pipe. 19: plastic recycling bins. 20: energy-eliminated orifice plate. 21: flow direction.

(4) The angular speed and the angle at time $t+\Delta t$ were calculated, which was written as follows:

$$
\begin{aligned}
\omega_{c}^{t+\Delta t} & =\left(\frac{M}{I}-\omega_{c} \times \omega_{c}\right) \Delta t, \\
\theta_{c}^{t+\Delta t} & =\frac{\left(\omega_{c}^{t}+\omega_{c}^{t+\Delta t}\right) \Delta t}{2}
\end{aligned}
$$

(5) Combined with the displacement $L_{c}^{t+\Delta t}$ and the angle $\theta_{c}^{t+\Delta t}$, the piped carriage moved to a new location by using the moving mesh technology, and then the meshes of the fluid domain were updated.

(6) The speed and the angular speed at time $t+\Delta t$ were regarded as the boundary conditions for the next iteration. These steps were repeated until the piped carriage arrived at the predefined locations.

\section{Materials and Methods}

4.1. Experimental System. To verify the reliability of simulated results from the commercial software ANSYS-Fluent
12.0 (http://www.ansys.com/Products/Fluids/ANSYS-Fluent), we conducted experimental investigations on the hydraulic characteristics of the piped carriage transport under different ratios $b$ within horizontal pipelines. The experimental system was composed of power devices, adjusting devices, conveying devices, and dropping and receiving devices, which was shown in Figure 7. The power devices comprised a centrifugal water pump and a water tank. The water tank was connected to the centrifugal pump by a steel penstock. The centrifugal water pump had a maximum operating water head of $32 \mathrm{~m}$, and the rated power of the pump motor was $15 \mathrm{~kW}$ at 2900 $\mathrm{rpm}$. The size of the water tank was $1.0 \mathrm{~m} \times 1.0 \mathrm{~m} \times 1.5 \mathrm{~m}$. Tracer particles inside the water tank were the hollow glass beads having the diameter in the range of about 50 to $100 \mu \mathrm{m}$, which were used to assist in measuring the flow velocity.

The adjusting devices were composed of an electromagnetic flowmeter, a regulating valve and a braking device. The electromagnetic flowmeter had an accuracy of $\pm 0.5 \%$ and a rated pressure of $0.6 \sim 4.0 \mathrm{MPa}$. The braking device was used to strictly control the start-up time for the piped carriage. Pipe fluid was adjusted using regulating valve to 

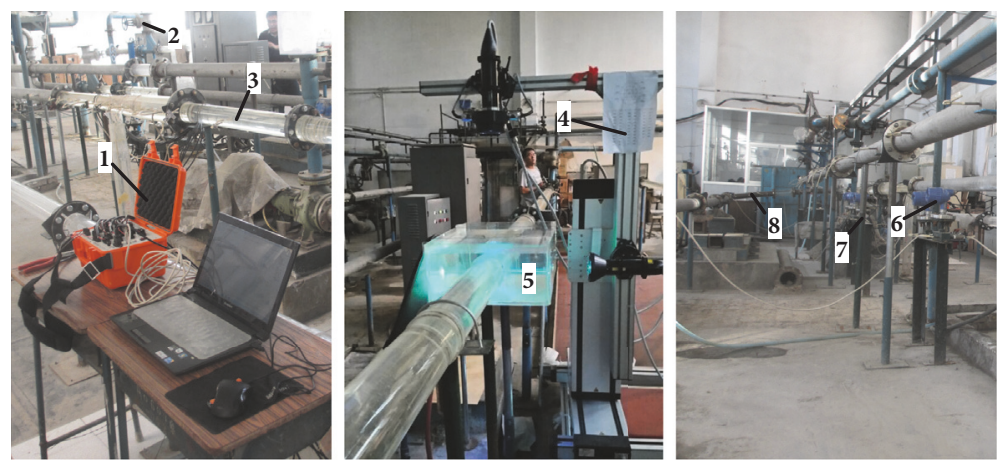

FIGURE 8: Experimental setup. Note: 1: standard dynamic pressure collection box. 2: input port for piped carriage. 3: horizontal pipe. 4: Laser Doppler Anemometry. 5: rectangular water channel. 6: pressure sensors. 7: iron holders. 8: $180^{\circ}$ pipe bend.

obtain the design flow of experimental schemes. Plexiglas pipes formed the conveying devices and were $28.62 \mathrm{~m}$ long with an inside diameter of $100 \mathrm{~mm}$ and a thickness of 5 $\mathrm{mm}$. The different pipes (A horizontal pipe and a $180^{\circ}$ pipe bend) were sealed and connected by round flanges and rubber gaskets. A rectangular water channel was located $2.2 \mathrm{~m}$ from the device of the piped carriage at the Plexiglas pipes, which was used to improve measurement precision by preventing refraction within the pipeline of the laser beam used to measure vehicle movement. The dropping and receiving devices consisted of an input port for the piped carriage, an energy-eliminated orifice plate, and plastic recycling bins. The energy-eliminated orifice plate was used to consume more energy for the high-velocity flow at the outlet piping. When the test was conducted, the water first was pumped into the steel penstocks from the water tank though the centrifugal water pump and then injected into the Plexiglas pipes by adjusting the electromagnetic flowmeter and the regulating valve. After the flow field became steady, the piped carriage was first fed into the piping systems through the input port for the piped carriage, then driven by the water though the pipelines, and finally collected in the plastic recycling bins at the end of the pipe, completing the travel process for the piped carriage. The water ultimately flowed back to the water tank again through the pipelines, which formed a closed primary loop.

4.2. Experiment Methods. In the study, twelve diameter ratios of the piped carriage were selected as the experimental variable, ranging from 0.4 to 0.95 with an interval of 0.05 . The diameter ratios had important implications: if the diameter ratio was less than 0.4 , the piped carriage would lose its capacity for freight, and if the diameter ratio was greater than 0.95 , the piped carriage would not move flexibly in the pipe bend sections. Therefore, in the experiment schemes the diameter ratio was in the range 0.4 to 0.95 , the length of the piped carriage was $0.1 \mathrm{~m}$, the transport loading that consisted of the weight of the bulk solid materials only and did not include the weight of the empty piped carriage itself was 0.6 $\mathrm{kg}$, and the pipe discharges were $40,50,60$, and $70 \mathrm{~m}^{3} \cdot \mathrm{h}^{-1}$, respectively.

The test measuring system comprised a Laser Doppler Anemometry, millisecond photoelectric timing devices, pressure sensors, a standard dynamic pressure collection box, and a high-speed camera, as shown in Figure 7. The Laser Doppler Anemometry manufactured by the Danish company Dantech and the millisecond photoelectric timing devices (702-3A, GOOD-STAR, China) were used to measure the velocity distributions of the flow field within the pipelines. The millisecond photoelectric timing devices measured the precise time when the piped carriage reached certain measuring cross-sections on the experimental system. The pressure sensors (BOOST, Su Zhou Xuan Sheng Medical Technology Co., Ltd., China) were linked to the standard dynamic pressure collection box (TST5103, Chengdu Test, China) to measure the piezometric heads along the pipelines. A highspeed camera (Memrecam GX-3, NAC, Japan) was mounted perpendicularly at the same height as the test section. The high-speed camera had an $1024 \times 1024$ image resolution and was connected to a personal computer via a RJ-45 connector twisted pair to measure the instantaneous speeds of the piped carriage at different times. Experimental setup was shown in Figure 8.

The test section had a total length of $5.8 \mathrm{~m}$ and was located in the middle of the horizontal sections in the experimental system, $2.7 \mathrm{~m}$ downstream from the inlet cross-section of the $180^{\circ}$ pipe bend, and $4.7 \mathrm{~m}$ upstream from the braking device of the piped carriage. In order to better verify the experimental results, the length of the test section was the same as that of the horizontal pipe model. Three different experimental cross-sections were used in the test section to measure velocity distributions of the flow field within the pipelines, in which $1^{\#}, 2^{\#}$, and $3^{\#}$ cross-sections were 2.6, 2.7, and $2.8 \mathrm{~m}$ from the inlet cross-section of the test section, respectively. There were 121 velocity measurement points in the experimental cross-sections, arranged at the intersection between the fifth concentric annuluses with $0.005 \mathrm{~m}$ intervals and the twelve pole axes at 30 degree intervals. There were 15 pressure measuring points on the right side of the horizontal wall of the test section, and the pressure measuring points were arranged at equal intervals of $0.5 \mathrm{~m}$. Measurements were made at each point three times and the average value of the measurements was reported. The two ends of the piped carriage in the flow direction were defined as the rear end (i.e., upstream end) and the front end (i.e., downstream 


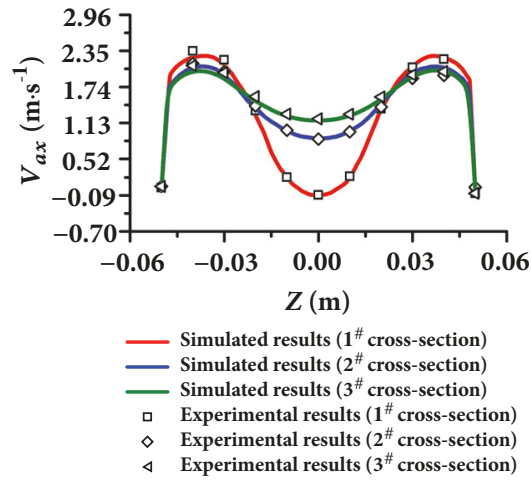

(a) $b=0.4$
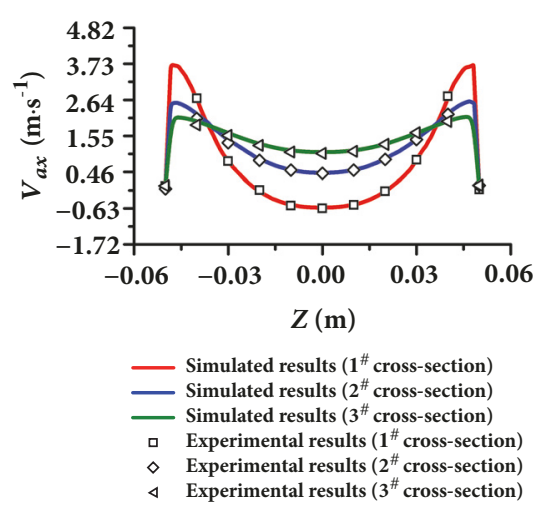

(d) $b=0.7$

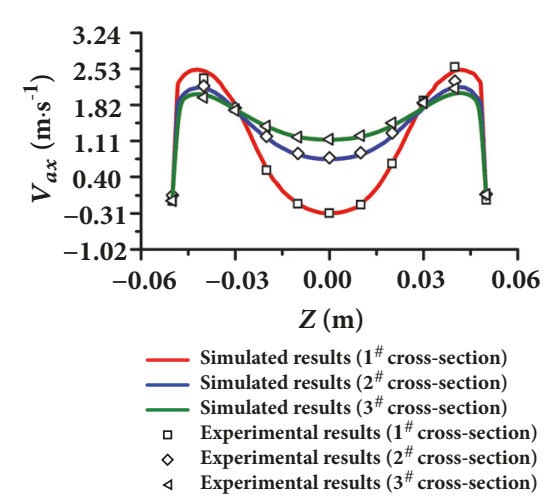

(b) $b=0.5$
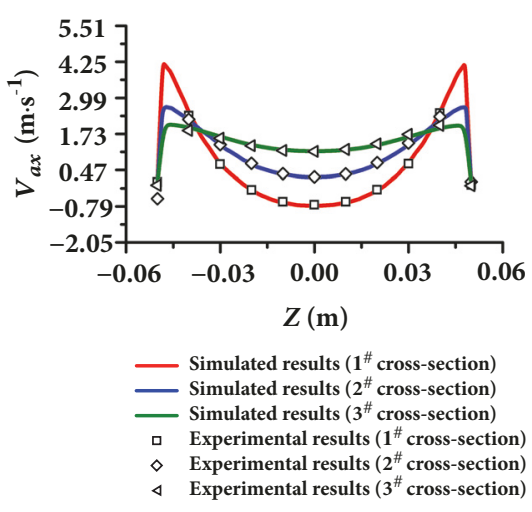

(e) $b=0.8$

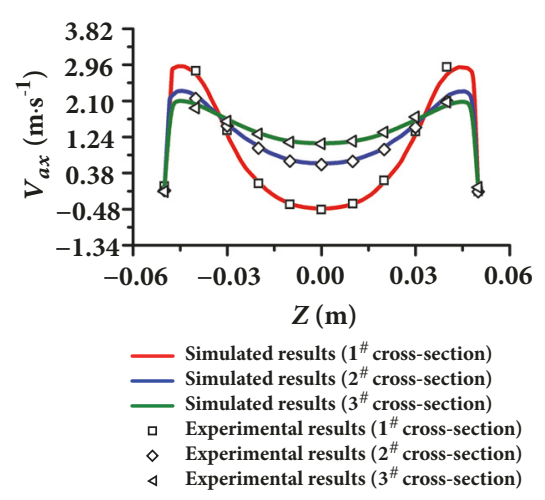

(c) $b=0.6$
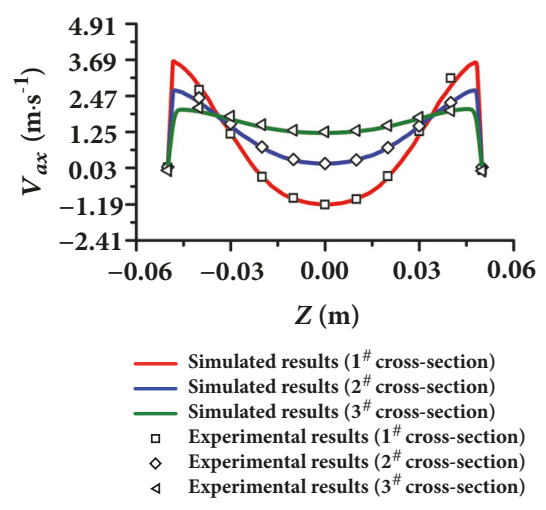

(f) $b=0.9$

FIGURE 9: Comparison of simulated and experimental results for axial velocity at horizontal position.

end), respectively. These terms were important in the analysis presented in Sections 5 and 6.

The velocity data of the different cross-sections and the pressure data along the pipelines were processed with Microsoft Office Excel 2013 and analyzed statistically with Suffer 11.0, Origin 9.0, and SPSS 21.0.

\section{Validation of Simulated Results}

Simulated results needed to be verified against the experimental results in order to gain confidence on these predictions. To further improve the reliability of the simulated results from ANSYS ${ }^{-}$Fluent 12.0, the simulated results and the experimental results were compared for three parameters: the velocity distributions of the three different cross-sections, the piezometric heads through the pipelines, and the instantaneous speeds of the piped carriage.

5.1. Velocity Distributions. Figure 9 showed the comparison of simulated and experimental results for the axial velocity at the horizontal position of the three different cross-sections when the center of the piped carriage with the six different diameter ratios arrived at the location that was $2.5 \mathrm{~m}$ from the inlet cross-section of the test section under the pipe discharge of $50 \mathrm{~m}^{3} \cdot \mathrm{h}^{-1}$.

From Figure 9 , the simulated axial velocity basically remained consistent with experimental results at the horizontal position of the three different cross-sections, and the maximum relative errors did not exceed $5.72 \%$. Because the piped carriage had great effects on the velocity distributions of flow field in the downstream pipe section, the experimental results of the velocity distributions at the downstream flow field of the piped carriage were analyzed by comparing them with the simulated results. There was obvious back-flow phenomena near the front end of the piped carriage. This was due to the fact that boundary layer separation was caused by the transition of the pipe fluid from the annular slit areas in the part where the piped carriage to the downstream flow field of the piped carriage, and resulting in the downstream fluid of the piped carriage to flow into the cavity areas. The closer experimental cross-sections were to the front end of the piped carriage, the more obvious the back-flow phenomena became. The reason was that the back-flow was caused by the boundary layer separation. Hence, the closer the experimental cross-sections were to the front end of the piped carriage, the more obvious effects of the boundary layer separation were.

5.2. Piezometric Heads. Figure 10 showed the comparison of simulated and experimental results for the piezometric heads through the pipelines when the center of the piped carriage with the six different diameter ratios arrived at the location that was $2.5 \mathrm{~m}$ from the inlet cross-section of the test section under the pipe discharge of $50 \mathrm{~m}^{3} \cdot \mathrm{h}^{-1}$. 


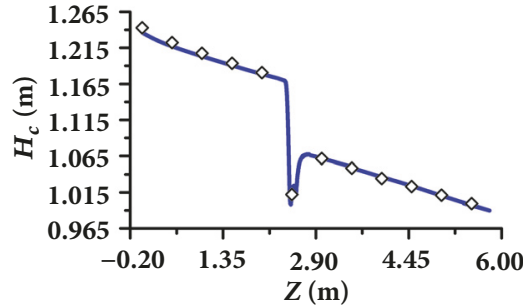

— Simulated results

$\diamond$ Experimental results

(a) $b=0.4$

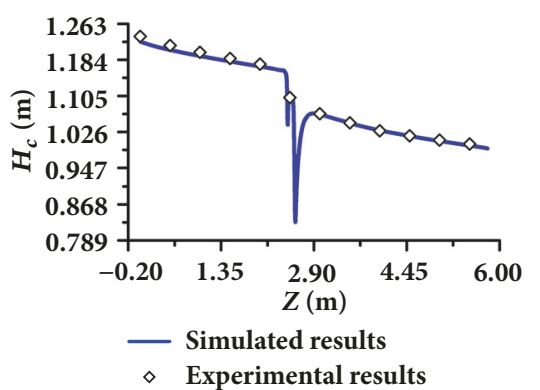

(d) $b=0.7$

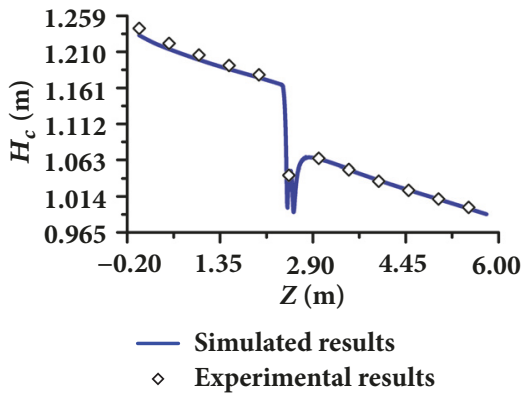

(b) $b=0.5$

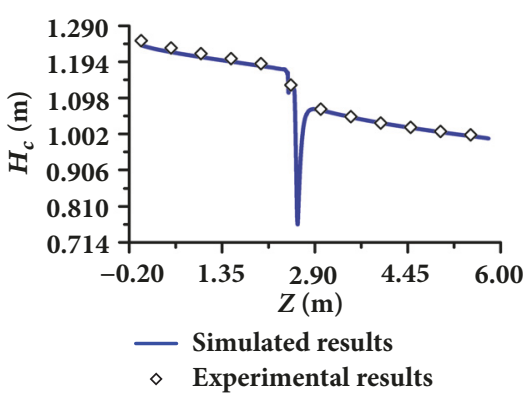

(e) $b=0.8$

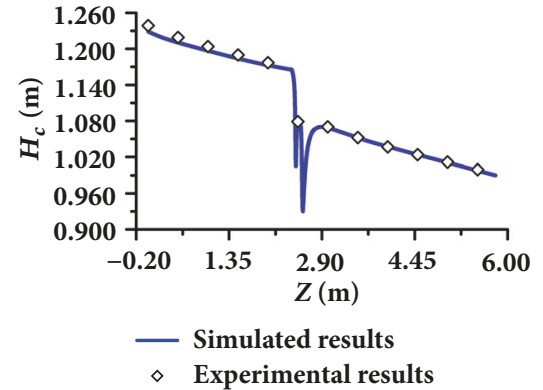

(c) $b=0.6$

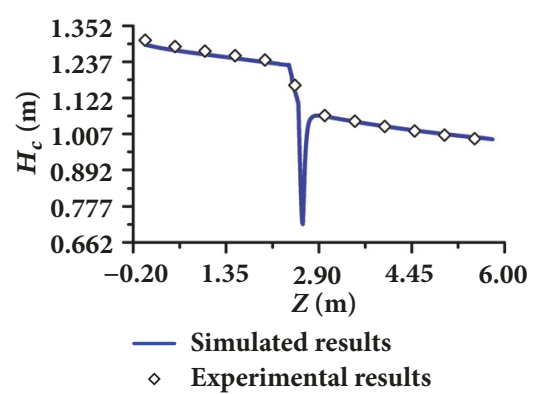

(f) $b=0.9$

FIGURE 10: Comparison of simulated and experimental results for piezometric heads through the pipeline.

From Figure 10, the simulated piezometric heads through the pipelines basically remained consistent with the experimental results, and the maximum relative errors did not exceed $1.58 \%$. As increasing diameter ratio, the pressure drop near the front end of the piped carriage gradually increased. This appeared because an increase of the back-flow areas near the front end of the piped carriage was caused by the increasing diameter ratio, thus leading to an increase in vortex energy losses. The distributions of the piezometric heads in the near-wall of the piped carriage showed W-type trend, which can be analyzed from perspective of energy losses. First of all, the support bodies caused a narrowing of cross-section and an increasing of flow velocity near the rear end of the piped carriage, resulting in a decrease in local pressure. The flow velocity then increased as the fluid entered the annular slit areas, causing a gradual increase in local pressure. Then, the local pressure decreased again due to boundary layer separation near the front end of the piped carriage. Finally, the kinetic energy of the annular slit flow was converted into the pressure energy of its downstream fluid so that the local pressure rose again in the downstream flow field of the piped carriage.

5.3. Instantaneous Speeds. Figure 11 showed the comparison of simulated results and experimental values for the instantaneous speeds when the piped carriage with the six different diameter ratios under the pipe discharge of $50 \mathrm{~m}^{3} \cdot \mathrm{h}^{-1}$ moved.

The instantaneous speeds of the piped carriage in Figure 11 were measured by a high-speed camera. The shooting frame rate of the high-speed camera was filmed at 500 images per second. From Figure 11, the simulated instantaneous speeds of the piped carriage basically remained consistent with the experimental results, and the maximum relative errors did not exceed $1.81 \%$. The irregular fluid pulsation pressure caused a fluctuating change in the instantaneous loadings that the fluid acted on the piped carriage and induced an enormous variation in the instantaneous acceleration of the piped carriage. As a result, this affected the instantaneous speed of the piped carriage to fluctuate within a certain subtle range. Due to the instantaneous speed fluctuations within the range of $\pm 1.5 \%$, it was considered that the motion status of the piped carriage in the horizontal pipelines kept the instantaneous speed in a relative constant state. The numerical simulation of the bidirectional fluidstructure interaction could be used to obtain the instantaneous speed of the piped carriage. The rotation speed of the piped carriage obtained from the numerical simulation and experimental measurement was smaller and negligible. Therefore, it can be considered that the piped carriage does not rotate around the $z$-axis within the pipeline and had only the translational speed along the $z$-axis. This approach had apparent advantages when compared with unidirectional fluid-structure interaction methods.

According to these comparisons, the commercial CFD software ANSYS-Fluent 12.0 was deemed to be suitable for determining the hydraulic characteristics of the piped carriage transported within the horizontal pipelines under different diameter ratios. The comparisons provided an important reference for the correctness of the simulated results presented subsequently in Section 6. 


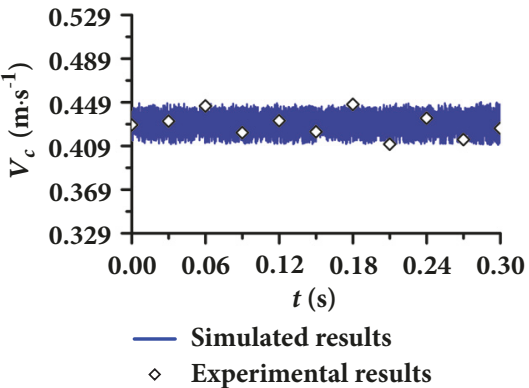

(a) $b=0.4 ; V_{b}=0.4296 \mathrm{~m} \cdot \mathrm{s}^{-1}$

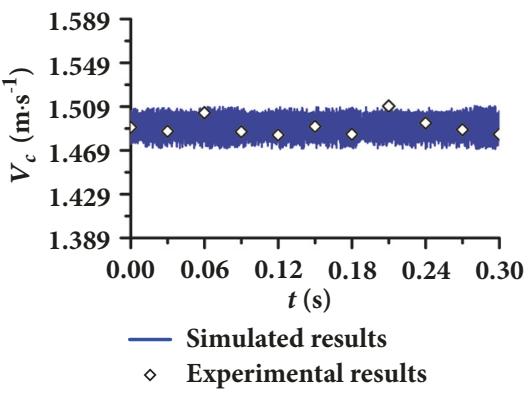

(d) $b=0.7 ; V_{b}=1.4905 \mathrm{~m} \cdot \mathrm{s}^{-1}$

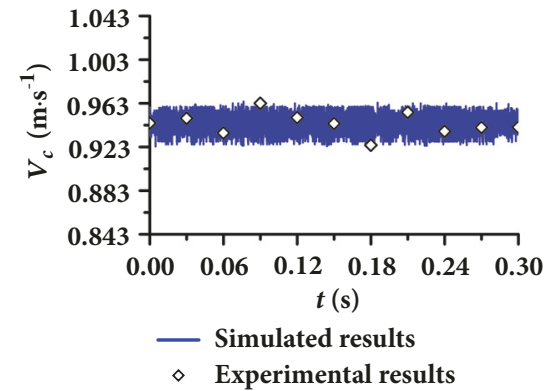

(b) $b=0.5 ; V_{b}=0.9439 \mathrm{~m} \cdot \mathrm{s}^{-1}$

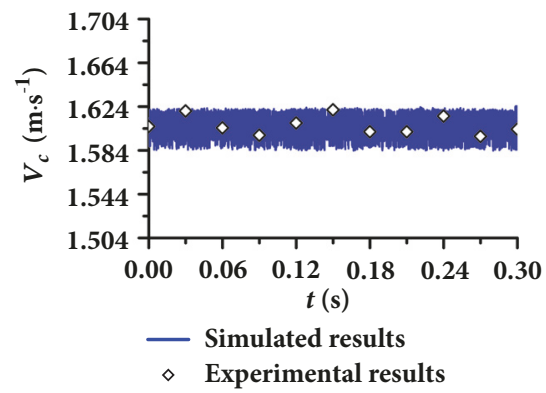

(e) $b=0.8 ; V_{b}=1.6053 \mathrm{~m} \cdot \mathrm{s}^{-1}$

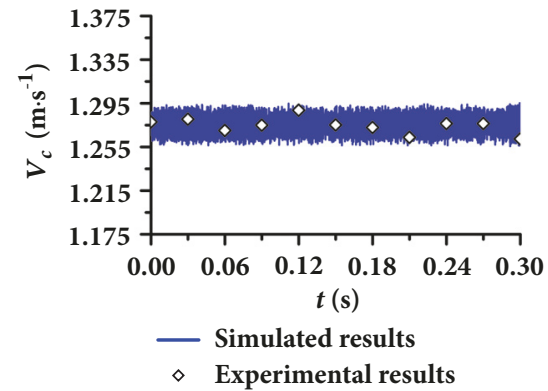

(c) $b=0.6 ; V_{b}=1.2758 \mathrm{~m} \cdot \mathrm{s}^{-1}$

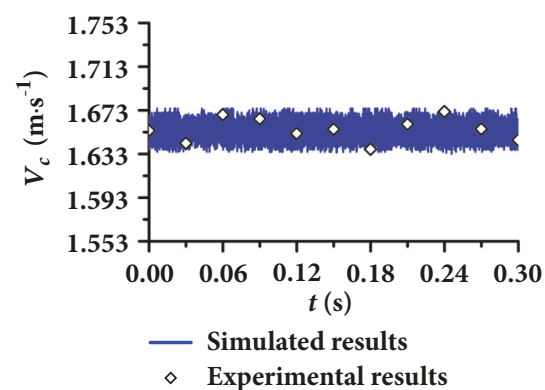

(f) $b=0.9 ; V_{b}=1.6544 \mathrm{~m} \cdot \mathrm{s}^{-1}$

FIGURE 11: Comparison of simulated results and experimental values for instantaneous speeds of a piped carriage.

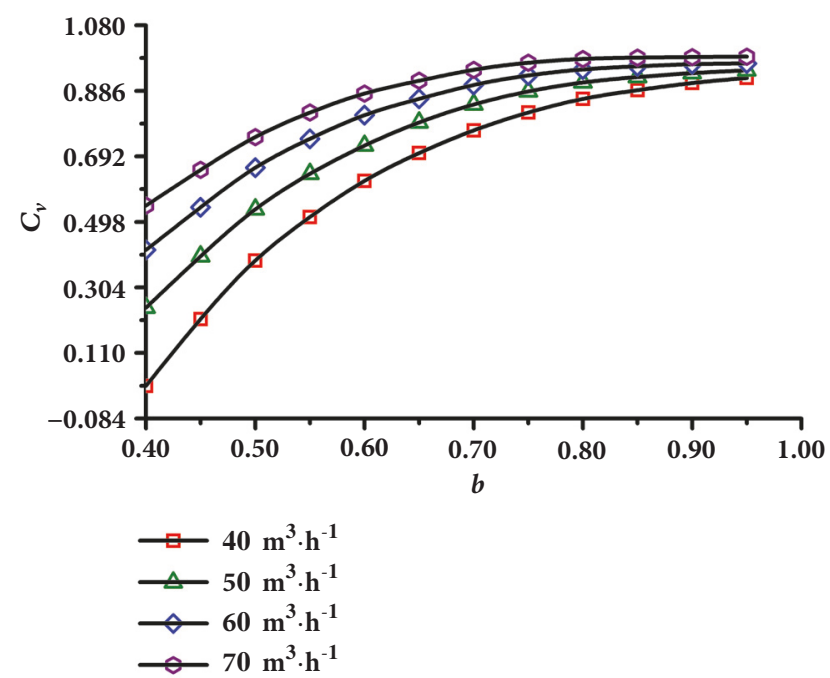

FIGURE 12: Relationship between average speed coefficient and diameter ratio for a piped carriage.

\section{Results and Discussion}

6.1. Speed Coefficient of Piped Carriage. Figure 12 showed the changes in the average speed coefficient as the diameter ratio increased. It has been observed that the average speed coefficient of the piped carriage gradually increased as the diameter ratio increased and was less than the average axial velocity of pipe fluid. The average axial velocity of the piped fluid was the ratio of the pipe discharge to the cross-sectional areas of the conveying pipes. Due to the pipe discharge of $50 \mathrm{~m}^{3} \cdot \mathrm{h}^{-1}$, the average axial velocity of the pipe fluid was calculated as $1.764 \mathrm{~m} \bullet \mathrm{s}^{-1}$. When the diameter ratio was less than 0.4 , the piped carriage was not suspended and pushed by the fluid within the pipelines. There was an interesting comparison between Figure 12 and Ellis's [5] research findings, relating to the cylindrical capsule with the density equal to that of its carrier fluid. The curves for the equal-density cylindrical capsule had a negative slope, showing a decreasing of the average speed coefficient with the diameter ratio, while in Figure 12, they generally had a positive slope. The densities of piped carriages involved in the manuscripts were greater than that of the carrier fluid and ranged from $1.845 \times 10^{3} \mathrm{~kg} \bullet \mathrm{m}^{-3}$ to $7.210 \times 10^{3} \mathrm{~kg} \bullet \mathrm{m}^{-3}$. When the density of the cylindrical capsule was equal to that of the carrier fluid, the average speed of the cylindrical capsule was closer to the axial velocity of the fluid in the center of the pipelines with the decreasing of the diameter ratio, which resulted in an increase in the average speed coefficient of the cylindrical capsule. When the density of the piped carriage was larger than that of the carrier fluid, both the normal pressure that the piped carriage acted on the bottom of the pipelines and the frictional resistance of the piped carriage experienced an increased trend with the decrease of the diameter ratio, leading to a decrease in the average speed coefficient of the piped carriage. For these reasons, a positive correlation existed between the diameter ratio and the average speed coefficient of the piped carriage with density larger than that of its carrier fluid. With the increase of the pipe discharge, the average speed coefficient of the piped carriage gradually increased. This was due to the fact that based on the law of momentum conservation, the relative velocity between the pipe fluid and the piped carriage 


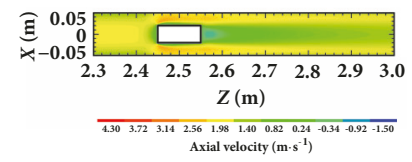

(a) $b=0.40 ; V_{b}=0.4296 \mathrm{~m} \cdot \mathrm{s}^{-1}$

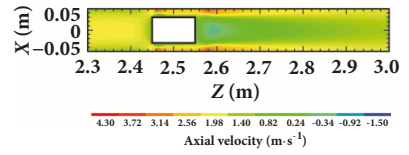

(e) $b=0.60 ; V_{b}=1.2758 \mathrm{~m} \cdot \mathrm{s}^{-1}$

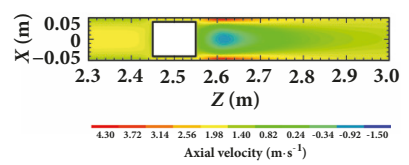

(i) $b=0.80 ; V_{b}=1.6053 \mathrm{~m} \cdot \mathrm{s}^{-1}$

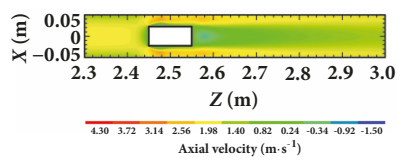

(b) $b=0.45 ; V_{b}=0.6982 \mathrm{~m} \cdot \mathrm{s}^{-1}$

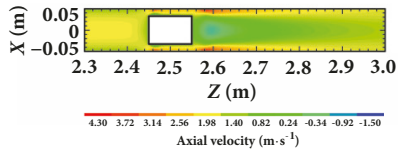

(f) $b=0.65 ; V_{b}=1.3971 \mathrm{~m} \cdot \mathrm{s}^{-1}$

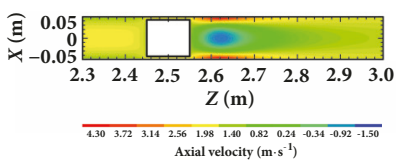

(j) $b=0.85 ; V_{b}=1.6335 \mathrm{~m} \cdot \mathrm{s}^{-1}$

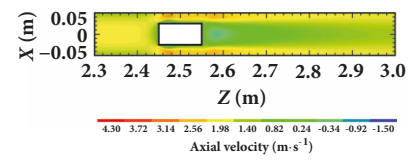

(c) $b=0.50 ; V_{b}=0.9439 \mathrm{~m} \cdot \mathrm{s}^{-1}$

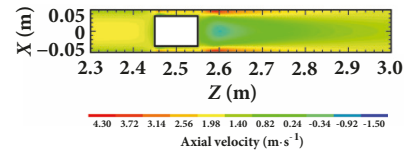

(g) $b=0.70 ; V_{b}=1.4905 \mathrm{~m} \cdot \mathrm{s}^{-1}$

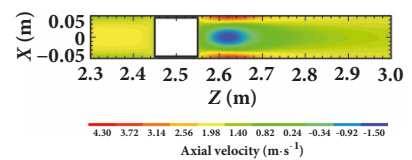

(k) $b=0.90 ; V_{b}=1.6544 \mathrm{~m} \cdot \mathrm{s}^{-1}$

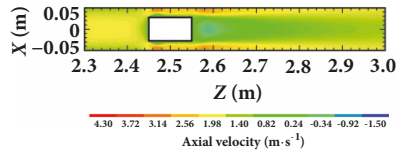

(d) $b=0.55 ; V_{b}=1.1287 \mathrm{~m} \cdot \mathrm{s}^{-1}$

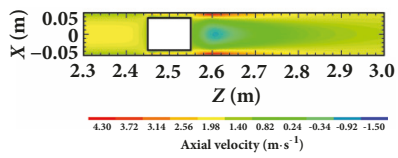

(h) $b=0.75 ; V_{b}=1.5588 \mathrm{~m} \cdot \mathrm{s}^{-1}$

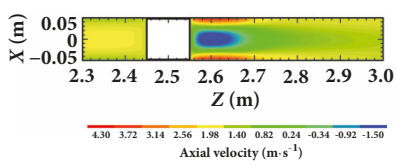

(l) $b=0.95 ; V_{b}=1.6687 \mathrm{~m} \cdot \mathrm{s}^{-1}$

FIGURE 13: Axial velocity distributions of transporting a piped carriage with different diameter ratios.

remained basically unchanged when the rolling frictional resistance of the piped carriage with different diameter ratios was constant. Hence, with the increasing of the pipe discharge, the average speed coefficient of the piped carriage also gradually increased. Meanwhile, the relationship between the average speed coefficient and the pipe discharge can also be further explained according to the motion model of the piped carriage. The increasing of the pipe discharge led to a gradual increase in $A_{3}$, which resulted in an increase in abscissa of intersection point between the parabola function (see (16) and (18)) and an x-axis, indicating that the average speed coefficient gradually increased with the increases of the pipe discharge.

6.2. Axial Velocity Distributions. Figure 13 showed the axial velocity distributions when the piped carriage with the different diameter ratios arrived at the location that was $2.5 \mathrm{~m}$ from the inlet cross-section of the test section under the pipe discharge of $50 \mathrm{~m}^{3} \cdot \mathrm{h}^{-1}$.

As shown in Figure 13, because the projection of the piped carriage onto the central horizontal cross-section of the pipeline was symmetrical around the $Z$ axis, the axial velocity distributions were more symmetrical. The obvious back-flow areas existed near the front end of the piped carriage, which appeared as cone-shaped distributions. The back-flow areas occurred because boundary layer separation was caused near the front end of the piped carriage by the adverse pressure gradient existed in the boundary layer areas of the piped carriage wall, resulting in the annular slit flow leaving the front end of the piped carriage, and the downstream fluid of the piped carriage randomly flowing into a cone-shaped cavity areas created by the boundary layer separation. There was a region of high axial velocity near the interior wall of the pipeline at the downstream flow field of the piped carriage. This appeared because the back-flow spread near the wall of the pipe at high velocity in the opposite direction when interacting with the front end of the piped carriage.
With increasing diameter ratio, the affected range in the axial velocity distributions of the back-flow areas at the front end of the piped carriage gradually increased. The phenomenon occurred because the back-flow areas mainly existed near the front end of the piped carriage, and the increasing diameter ratio led to an increase in the crosssectional areas of the front end of the piped carriage, thus producing even more back-flow areas. As the diameter ratio increased, the affected range of the axial velocity distributions near the rear end of the piped carriage gradually decreased. The reason for this was that the speed of the piped carriage was closer to the average axial velocity of the pipe fluid with the increasing diameter ratio. As a result, the disturbed areas caused with the front end of the piped carriage became smaller.

Figure 14 showed the axial velocity distributions of transporting a piped carriage having a diameter ratio of 0.6 at different times under the pipe discharge of $50 \mathrm{~m}^{3} \cdot \mathrm{h}^{-1}$.

Due to the interaction between the piped carriage and the pipe fluid, the axial velocity distributions at the downstream flow field of the piped carriage appeared to be unsteady. However, as can be seen from Figure 14, the axial velocity distributions of the horizontal section within the pipelines transporting the piped carriage at different times were the same, and there was no periodic change of the axial velocity distributions over time. The difference between the maximum axial velocity at different times and the average maximum axial velocity did not exceed $1.93 \%$. This appeared because instantaneous speed of the piped carriage changed within a limited range, leading to a small change in the unsteady characteristics of the axial velocity distributions of the bidirectional interaction system formed by the piped carriage and the pipe fluid at any time.

6.3. Radial Velocity Distributions. Figure 15 showed the radial velocity distributions when the piped carriage with the different diameter ratios arrived at the location that was $2.5 \mathrm{~m}$ 


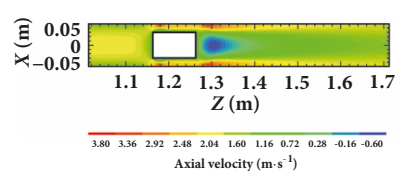

(a) $t=0.4 \mathrm{~s} ; S_{c}=1.213 \mathrm{~m} ; V_{c}=$ $1.2779 \mathrm{~m} \cdot \mathrm{s}^{-1}$

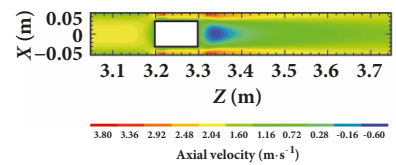

(e) $t=2.0 \mathrm{~s} ; S_{c}=3.248 \mathrm{~m} ; V_{c}=$ $1.2867 \mathrm{~m} \cdot \mathrm{s}^{-1}$

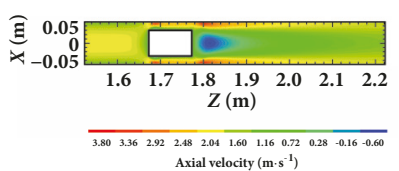

(b) $t=0.8 \mathrm{~s} ; S_{c}=1.723 \mathrm{~m} ; V_{c}=$ $1.2652 \mathrm{~m} \cdot \mathrm{s}^{-1}$

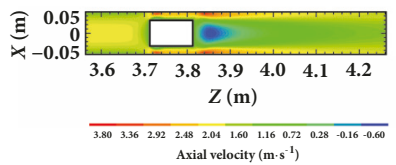

(f) $t=2.4 \mathrm{~s} ; S_{c}=3.763 \mathrm{~m} ; V_{c}=$ $1.2685 \mathrm{~m} \cdot \mathrm{s}^{-1}$

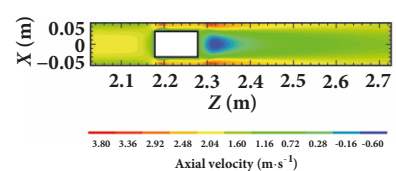

(c) $t=1.2 \mathrm{~s} ; S_{c}=2.228 \mathrm{~m} ; V_{c}=$ $1.2812 \mathrm{~m} \cdot \mathrm{s}^{-1}$

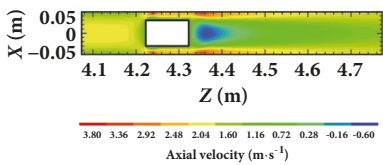

(g) $t=2.8 \mathrm{~s} ; S_{c}=4.273 \mathrm{~m} ; V_{c}=$ $1.2836 \mathrm{~m} \cdot \mathrm{s}^{-1}$

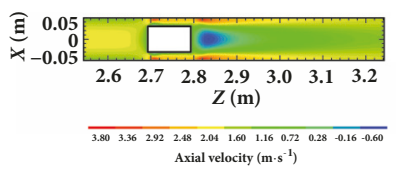

(d) $t=1.6 \mathrm{~s} ; S_{c}=2.743 \mathrm{~m} ; V_{c}=$ $1.2753 \mathrm{~m} \cdot \mathrm{s}^{-1}$

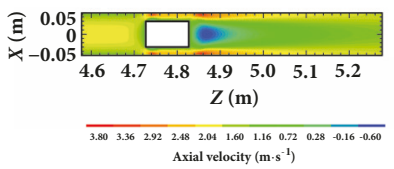

(h) $t=3.2 \mathrm{~s} ; S_{c}=4.777 \mathrm{~m} ; V_{c}=$ $1.2723 \mathrm{~m} \cdot \mathrm{s}^{-1}$

FIGURE 14: Axial velocity distributions of transporting a piped carriage at different times. Note: $S_{c}$ represents distance from inlet crosssection of geometric model to center of piped carriage.

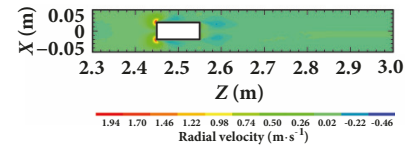

(a) $b=0.40 ; V_{b}=0.4296 \mathrm{~m} \cdot \mathrm{s}^{-1}$

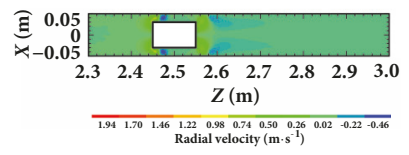

(e) $b=0.60 ; V_{b}=1.2758 \mathrm{~m} \cdot \mathrm{s}^{-1}$

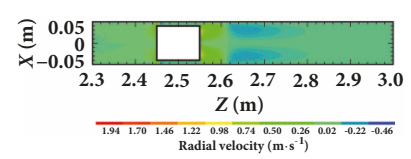

(i) $b=0.80 ; V_{b}=1.6053 \mathrm{~m} \cdot \mathrm{s}^{-1}$

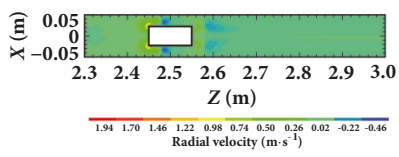

(b) $b=0.45 ; V_{b}=0.6982 \mathrm{~m} \cdot \mathrm{s}^{-1}$

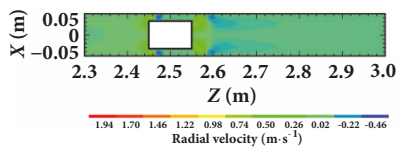

(f) $b=0.65 ; V_{b}=1.3971 \mathrm{~m} \cdot \mathrm{s}^{-1}$

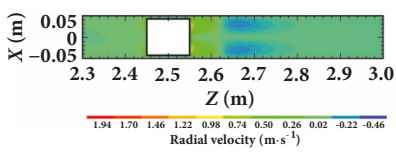

(j) $b=0.85 ; V_{b}=1.6335 \mathrm{~m} \cdot \mathrm{s}^{-1}$

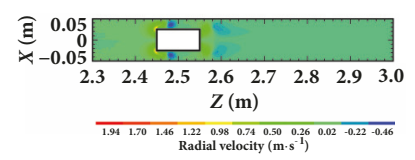

(c) $b=0.50 ; V_{b}=0.9439 \mathrm{~m} \cdot \mathrm{s}^{-1}$

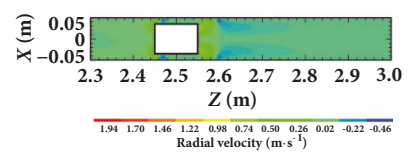

(g) $b=0.70 ; V_{b}=1.4905 \mathrm{~m} \cdot \mathrm{s}^{-1}$

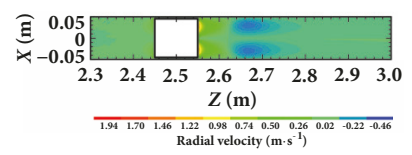

(k) $b=0.90 ; V_{b}=1.6544 \mathrm{~m} \cdot \mathrm{s}^{-1}$

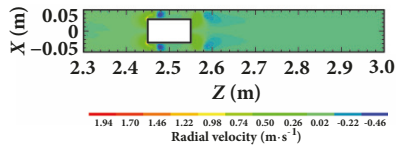

(d) $b=0.55 ; V_{b}=1.1287 \mathrm{~m} \cdot \mathrm{s}^{-1}$

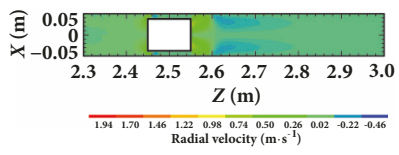

(h) $b=0.75 ; V_{b}=1.5588 \mathrm{~m} \cdot \mathrm{s}^{-1}$

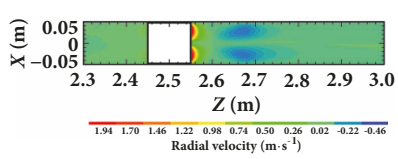

(l) $b=0.95 ; V_{b}=1.6687 \mathrm{~m} \cdot \mathrm{s}^{-1}$

FIGURE 15: Radial velocity distributions of transporting a piped carriage with different diameter ratios.

from the inlet cross-section of the test section under the pipe discharge of $50 \mathrm{~m}^{3} \cdot \mathrm{h}^{-1}$.

As shown in Figure 15, because the projection of the piped carriage onto the central horizontal cross-section of the pipeline was symmetrical around the $Z$ axis, the radial velocity distributions were more symmetrical. The radial velocity of the flow field within the pipelines was mainly distributed near the front and rear end of the piped carriage, and the direction of the radial velocity dispersed from the pipe center towards the pipe wall. This behavior occurred for different reasons at the front and rear end of the piped carriage. Regarding the radial velocity near the rear end of the piped carriage, the reason was that the pipe fluid affected by the rear end of the piped carriage decreased towards the areas of the annular slit flow, leading to more obvious radial velocity distributions. As for the radial velocity near the front end of the piped carriage, the reason was that the back-flow interacted with the front end of the piped carriage, resulting in the formation of the radial velocity distributions. These influences caused the outward diffusion of the radial velocity.
With the increase of relative velocity between the pipe fluid and the piped carriage, the radial velocity gradually increased. As the relative velocity between the front end of the piped carriage and the pipe fluid increased gradually, the radial velocity near the front end of the piped carriage showed a tendency to increase. The radial velocity at the downstream flow field of the piped carriage was low and the flow direction was from the wall of pipe to the center of the pipe; this was caused by the diffusion of the annular slit flow towards the downstream flow field of the piped carriage. With the increase of the diameter ratio, the affected areas of the radial velocity near the front end of the piped carriage gradually increased, while the affected areas of the radial velocity near the rear of the piped carriage gradually decreased. These results occurred because as the diameter ratio increased, the increasing relative velocity of both the front end of the piped carriage and the pipe fluid led to an increase in the affected areas of the radial velocity. Simultaneously, the decreasing relative velocity of both the rear end of the piped carriage and the pipe fluid decreased the areas of affected radial velocity. 


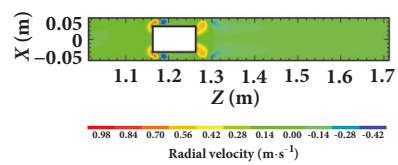

(a) $t=0.4 \mathrm{~s} ; S_{c}=1.213 \mathrm{~m} ; V_{c}=$ $1.2779 \mathrm{~m} \cdot \mathrm{s}^{-1}$

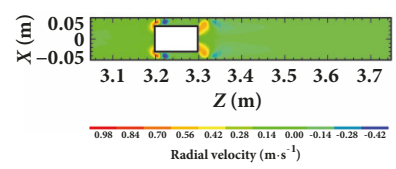

(e) $t=2.0 \mathrm{~s} ; S_{c}=3.248 \mathrm{~m} ; V_{c}=$ $1.2867 \mathrm{~m} \cdot \mathrm{s}^{-1}$

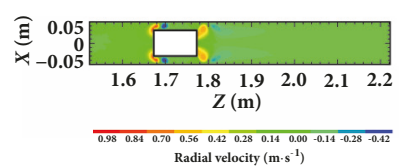

(b) $t=0.8 \mathrm{~s} ; S_{c}=1.723 \mathrm{~m} ; V_{c}=$ $1.2652 \mathrm{~m} \cdot \mathrm{s}^{-1}$

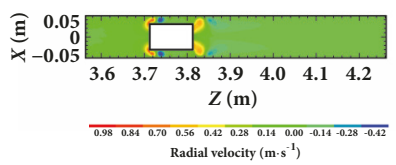
(f) $t=2.4 \mathrm{~s} ; S_{c}=3.763 \mathrm{~m} ; V_{c}=$
$1.2685 \mathrm{~m} \cdot \mathrm{s}^{-1}$

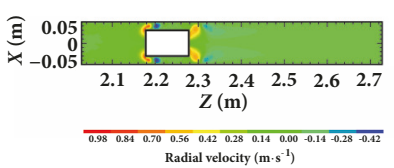

(c) $t=1.2 \mathrm{~s} ; S_{c}=2.228 \mathrm{~m} ; V_{c}=$ $1.2812 \mathrm{~m} \cdot \mathrm{s}^{-1}$

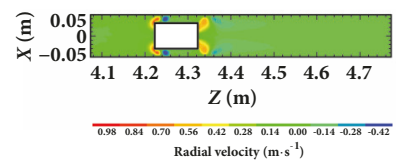

(g) $t=2.8 \mathrm{~s} ; S_{c}=4.273 \mathrm{~m} ; V_{c}=$ $1.2836 \mathrm{~m} \cdot \mathrm{s}^{-1}$

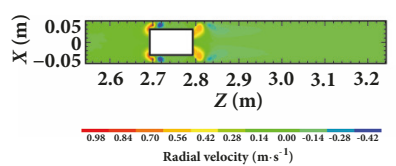

(d) $t=1.6 \mathrm{~s} ; S_{c}=2.743 \mathrm{~m} ; V_{c}=$ $1.2753 \mathrm{~m} \cdot \mathrm{s}^{-1}$

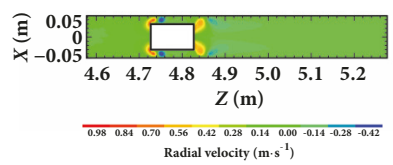

(h) $t=3.2 \mathrm{~s} ; S_{c}=4.777 \mathrm{~m} ; V_{c}=$ $1.2723 \mathrm{~m} \cdot \mathrm{s}^{-1}$

FIGURE 16: Radial velocity distributions of transporting a piped carriage at different times.

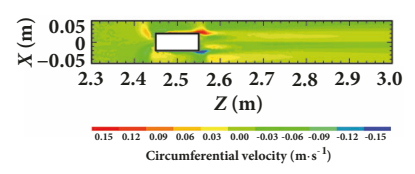

(a) $b=0.40 ; V_{b}=0.4296 \mathrm{~m} \cdot \mathrm{s}^{-1}$

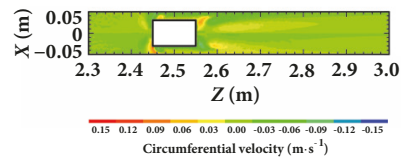

(e) $b=0.60 ; V_{b}=1.2758 \mathrm{~m} \cdot \mathrm{s}^{-1}$

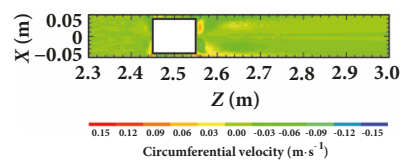

(i) $b=0.80 ; V_{b}=1.6053 \mathrm{~m} \cdot \mathrm{s}^{-1}$

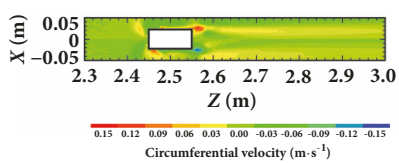

(b) $b=0.45 ; V_{b}=0.6982 \mathrm{~m} \cdot \mathrm{s}^{-1}$

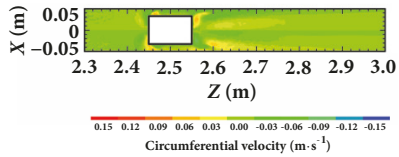

(f) $b=0.65 ; V_{b}=1.3971 \mathrm{~m} \cdot \mathrm{s}^{-1}$

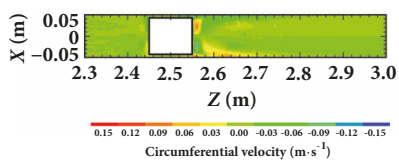

(j) $b=0.85 ; V_{b}=1.6335 \mathrm{~m} \cdot \mathrm{s}^{-1}$

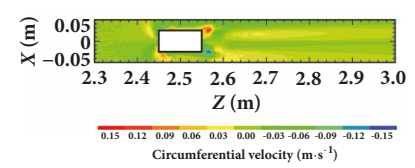

(c) $b=0.50 ; V_{b}=0.9439 \mathrm{~m} \cdot \mathrm{s}^{-1}$

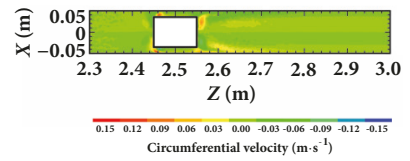

(g) $b=0.70 ; V_{b}=1.4905 \mathrm{~m} \cdot \mathrm{s}^{-1}$

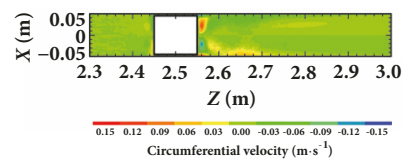

(k) $b=0.90 ; V_{b}=1.6544 \mathrm{~m} \cdot \mathrm{s}^{-1}$

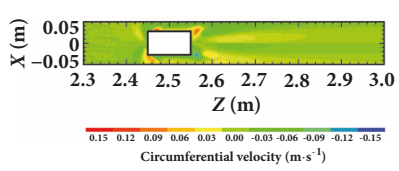

(d) $b=0.55 ; V_{b}=1.1287 \mathrm{~m} \cdot \mathrm{s}^{-1}$

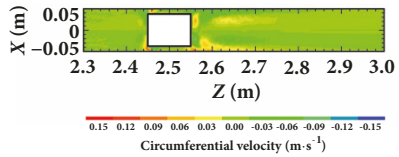

(h) $b=0.75 ; V_{b}=1.5588 \mathrm{~m} \cdot \mathrm{s}^{-1}$

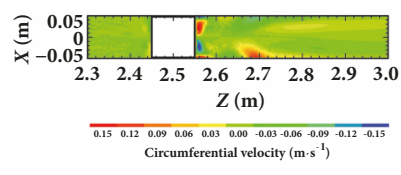

(l) $b=0.95 ; V_{b}=1.6687 \mathrm{~m} \cdot \mathrm{s}^{-1}$

FIGURE 17: Circumferential velocity distributions of transporting a piped carriage with different diameter ratios.

The radial flow velocity changed cyclically when the pipe fluid spread towards the downstream flow field of the piped carriage. Hence, it was shown that there was an alternating of the forward and reverse radial velocity at the downstream flow field of the piped carriage.

Figure 16 showed the radial velocity distributions of transporting a piped carriage having a diameter ratio of 0.6 at different times under the pipe discharge of $50 \mathrm{~m}^{3} \cdot \mathrm{h}^{-1}$.

Due to the interaction between the piped carriage and the pipe fluid, the radial velocity distributions at the downstream flow field of the piped carriage appeared to be unsteady. However, as can be seen from Figure 16, the radial velocity distributions of the horizontal section within the pipelines transporting the piped carriage at different times were the same. There was no periodic change of the radial velocity distributions over time. The difference between the maximum radial velocity at different times and the average maximum radial velocity did not exceed $0.97 \%$. This appeared because the instantaneous speed of the piped carriage changed within a limited range, leading to a small change in the unsteady characteristics of the radial velocity distributions of the bidirectional interaction system formed by the piped carriage and the pipe fluid at any time.

6.4. Circumferential Velocity Distributions. Figure 17 showed the circumferential velocity distributions when the piped carriage with the different diameter ratios arrived at the location that was $2.5 \mathrm{~m}$ from the inlet cross-section of the test section under the pipe discharge of $50 \mathrm{~m}^{3} \cdot \mathrm{h}^{-1}$.

As shown in Figure 17, the circumferential velocity magnitude distributions inside the pipeline are symmetric around the $Z$ axis, while circumferential velocity distributions of both the positive and negative $x$-axis areas occurred in the opposite direction. The direction of the circumferential velocity was counterclockwise in the positive $x$-axis areas and clockwise in the negative $x$-axis areas. The circumferential velocity near the front end of the piped carriage was mainly caused by the support bodies, thus the maximum circumferential velocity appeared near the wall of the supports. The greater was the relative velocity between the pipe fluid and the piped carriage, the larger was the circumferential velocity of the pipe fluid. The circumferential velocity near the front end of the piped carriage was thought to be higher than that near the rear 


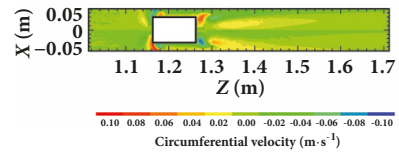

(a) $t=0.4 \mathrm{~s} ; S_{c}=1.213 \mathrm{~m} ; V_{c}=$ $1.2779 \mathrm{~m} \cdot \mathrm{s}^{-1}$

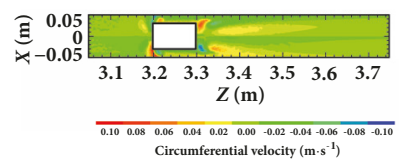

(e) $t=2.0 \mathrm{~s} ; S_{c}=3.248 \mathrm{~m} ; V_{c}=$ $1.2867 \mathrm{~m} \cdot \mathrm{s}^{-1}$

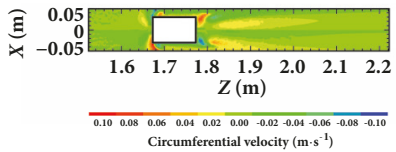

(b) $t=0.8 \mathrm{~s} ; S_{c}=1.723 \mathrm{~m} ; V_{c}=$ $1.2652 \mathrm{~m} \cdot \mathrm{s}^{-1}$

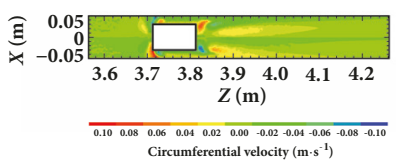

(f) $t=2.4 \mathrm{~s} ; S_{c}=3.763 \mathrm{~m} ; V_{c}=$ $1.2685 \mathrm{~m} \cdot \mathrm{s}^{-1}$

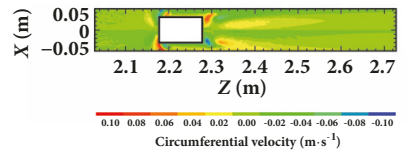

(c) $t=1.2 \mathrm{~s} ; S_{c}=2.228 \mathrm{~m} ; V_{c}=$ $1.2812 \mathrm{~m} \cdot \mathrm{s}^{-1}$

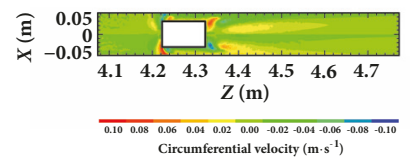

(g) $t=2.8 \mathrm{~s} ; S_{c}=4.273 \mathrm{~m} ; V_{c}=$ $1.2836 \mathrm{~m} \cdot \mathrm{s}^{-1}$

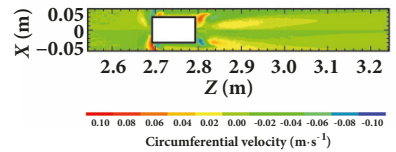

(d) $t=1.6 \mathrm{~s} ; S_{c}=2.743 \mathrm{~m} ; V_{c}=$ $1.2753 \mathrm{~m} \cdot \mathrm{s}^{-1}$

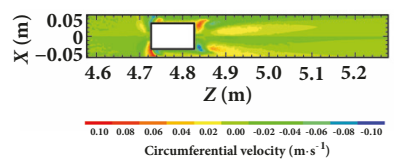

(h) $t=3.2 \mathrm{~s} ; S_{c}=4.777 \mathrm{~m} ; V_{c}=$ $1.2723 \mathrm{~m} \cdot \mathrm{s}^{-1}$

FIGURE 18: Circumferential velocity distributions of transporting a piped carriage at different times.

end of the piped carriage because there was a larger relative velocity between the front end of the piped carriage and the pipe fluid. The circumferential velocity of both back-flow near the front end of the piped carriage and the annular slit flow around the support bodies occurred in the same directions, although the axial velocity of these two flows occurred in opposite directions. The circumferential velocity at the downstream flow field of the piped carriage exhibited a periodic fish-scale type distributions, which was mainly the result of the diffusion of the circumferential velocity at the downstream flow field of the piped carriage.

As diameter ratio increased, the affected areas of the circumferential velocity near the front end of the piped carriage gradually first decreased and then increased. This variation occurred because when the diameter ratio was less than 0.75 , the circumferential velocity near the front of the piped carriage was mainly formed by the annular slit flow around the support bodies. The increasing diameter ratio caused an increasing speed of the piped carriage and a decreasing relative velocity between the support bodies and the annular slit flow, leading to decreasing trends of both the circumferential velocity and its affected areas near the front of the piped carriage. In addition, when the diameter ratio was greater than 0.75 , the circumferential velocity near the front of the piped carriage was mainly formed by the backflow around the support bodies. The increasing diameter ratio caused an increasing relative velocity between the support bodies and the back-flow near the front of the piped carriage, leading to increasing trends of both the circumferential velocity and its affected areas near the front of the piped carriage. According to these observations, the energy loss at the downstream flow field of the piped carriage was projected to gradually increase when the diameter ratio exceeded 0.75 (a result confirmed subsequently in Section 6.7).

Figure 18 showed the circumferential velocity distributions of transporting a piped carriage having a diameter ratio of 0.6 at different times under the pipe discharge of $50 \mathrm{~m}^{3} \cdot \mathrm{h}^{-1}$.

Due to the interaction between the piped carriage and the pipe fluid, the circumferential velocity distributions at the downstream flow field of the piped carriage appeared to be unsteady. However, as can be seen from Figure 18, the circumferential velocity distributions of the horizontal section within the pipelines transporting the piped carriage at different times were the same. There was no periodic change of the circumferential velocity distributions over time. The difference between the maximum circumferential velocity at different times and the average maximum circumferential velocity did not exceed $1.72 \%$. This appeared because the instantaneous speed of the piped carriage changed within a limited range, leading to a small change in the unsteady characteristics of the circumferential velocity distributions of the bidirectional interaction system formed by the piped carriage and the pipe fluid at any time.

6.5. Pressure Distributions. Figure 19 showed the pressure distributions when the piped carriage with the different diameter ratios arrived at the location that was $2.5 \mathrm{~m}$ from the inlet cross-section of the test section under the pipe discharge of $50 \mathrm{~m}^{3} \cdot \mathrm{h}^{-1}$.

As shown in Figure 19, because the projection of the piped carriage onto the central horizontal cross-section of the pipeline was symmetrical around the $Z$ axis, the pressure distributions were more symmetrical. Local high pressure areas of the flow field within the pipeline existed near the front and rear ends of the piped carriage. These areas developed because the fluid kinetic energy near the rear end of the piped carriage was converted into pressure energy under the action of the piped carriage. Simultaneously, interaction between the front end of the piped carriage and the local back-flow caused the kinetic energy of the pipe fluid to be converted into pressure energy again. The greater was the relative velocity of the piped carriage and the pipe fluid, the greater was the local pressure growth. Thus the pressure growth at the front end of the piped carriage was higher than that at the rear end.

An obvious low pressure areas was developed at the downstream flow field of the piped carriage, but the pressure at the downstream flow field of this area gradually increased through the pipeline. This occurred because when the annular slit flow mixed with the downstream fluid of the piped carriage, the flow field within the pipelines underwent two processes: energy dissipation and energy conversion. When the annular slit flow collided with the downstream fluid of the piped carriage, kinetic energy was converted into the internal energy of the fluid because of the viscosity of the fluid 


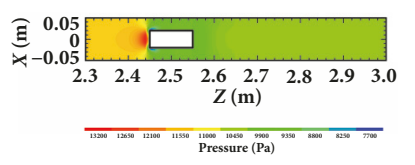

(a) $b=0.40 ; V_{b}=0.4296 \mathrm{~m} \cdot \mathrm{s}^{-1}$

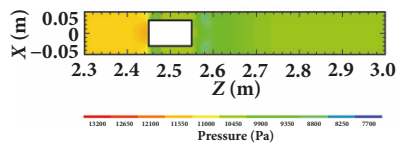

(e) $b=0.60 ; V_{b}=1.2758 \mathrm{~m} \cdot \mathrm{s}^{-1}$

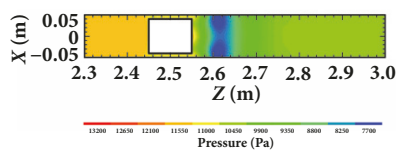

(i) $b=0.80 ; V_{b}=1.6053 \mathrm{~m} \cdot \mathrm{s}^{-1}$

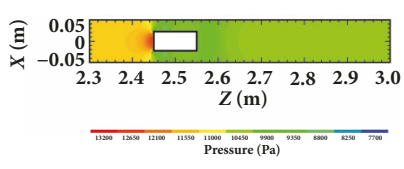

(b) $b=0.45 ; V_{b}=0.6982 \mathrm{~m} \cdot \mathrm{s}^{-1}$

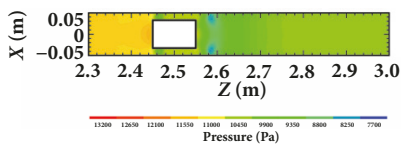

(f) $b=0.65 ; V_{b}=1.3971 \mathrm{~m} \cdot \mathrm{s}^{-1}$

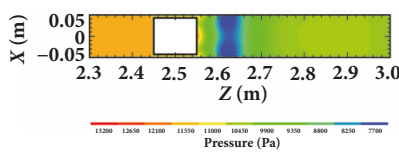

(j) $b=0.85 ; V_{b}=1.6335 \mathrm{~m} \cdot \mathrm{s}^{-1}$

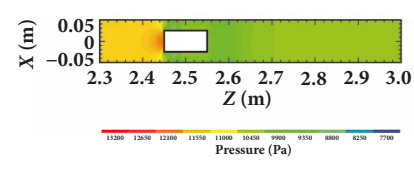

(c) $b=0.50 ; V_{b}=0.9439 \mathrm{~m} \cdot \mathrm{s}^{-1}$

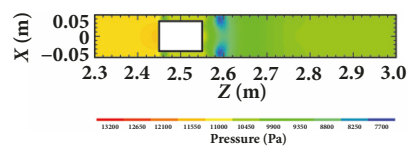

(g) $b=0.70 ; V_{b}=1.4905 \mathrm{~m} \cdot \mathrm{s}^{-1}$

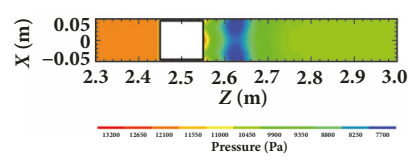

(k) $b=0.90 ; V_{b}=1.6544 \mathrm{~m} \cdot \mathrm{s}^{-1}$

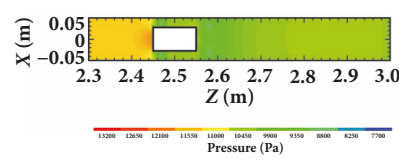

(d) $b=0.55 ; V_{b}=1.1287 \mathrm{~m} \cdot \mathrm{s}^{-1}$

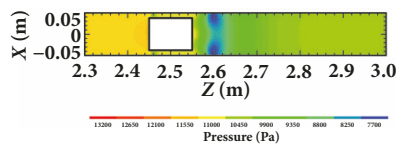

(h) $b=0.75 ; V_{b}=1.5588 \mathrm{~m} \cdot \mathrm{s}^{-1}$

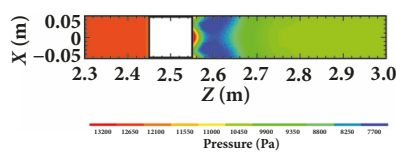

(l) $b=0.95 ; V_{b}=1.6687 \mathrm{~m} \cdot \mathrm{s}^{-1}$

FIGURE 19: Pressure distributions of transporting a piped carriage with different diameter ratios.

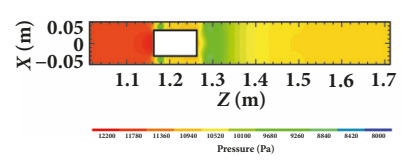

(a) $t=0.4 \mathrm{~s} ; S_{c}=1.213 \mathrm{~m} ; V_{c}=$ $1.2779 \mathrm{~m} \cdot \mathrm{s}^{-1}$

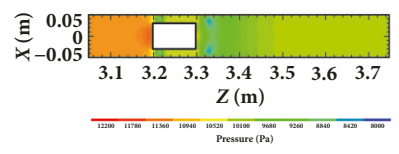

(e) $t=2.0 \mathrm{~s} ; S_{c}=3.248 \mathrm{~m} ; V_{c}=$ $1.2867 \mathrm{~m} \cdot \mathrm{s}^{-1}$

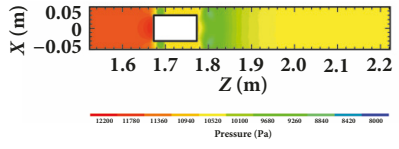

(b) $t=0.8 \mathrm{~s} ; S_{c}=1.723 \mathrm{~m} ; V_{c}=$ $1.2652 \mathrm{~m} \cdot \mathrm{s}^{-1}$

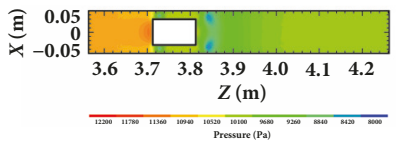

(f) $t=2.4 \mathrm{~s} ; S_{c}=3.763 \mathrm{~m} ; V_{c}=$ $1.2685 \mathrm{~m} \cdot \mathrm{s}^{-1}$

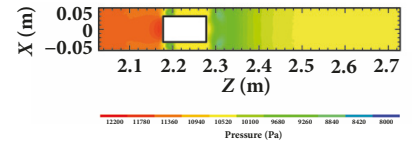

(c) $t=1.2 \mathrm{~s} ; S_{c}=2.228 \mathrm{~m} ; V_{c}=$ $1.2812 \mathrm{~m} \cdot \mathrm{s}^{-1}$

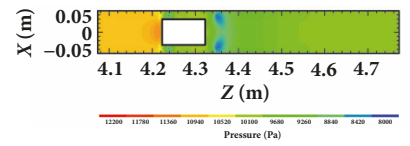

(g) $t=2.8 \mathrm{~s} ; S_{c}=4.273 \mathrm{~m} ; V_{c}=$ $1.2836 \mathrm{~m} \cdot \mathrm{s}^{-1}$

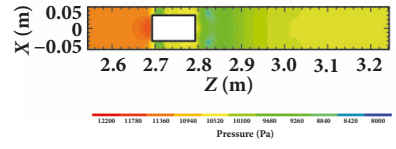

(d) $t=1.6 \mathrm{~s} ; S_{c}=2.743 \mathrm{~m} ; V_{c}=$ $1.2753 \mathrm{~m} \cdot \mathrm{s}^{-1}$

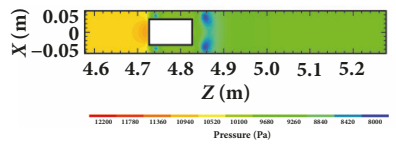

(h) $t=3.2 \mathrm{~s} ; S_{c}=4.777 \mathrm{~m} ; V_{c}=$ $1.2723 \mathrm{~m} \cdot \mathrm{s}^{-1}$

Figure 20: Pressure distributions of transporting a piped carriage at different times.

(energy dissipation). In contrast, energy conversion occurred when kinetic energy of the annular slit flow was converted to pressure energy of the downstream fluid. The dual effects of energy dissipation and energy conversion together caused the local low pressure areas in the downstream flow field of the piped carriage, while the energy conversion caused the pressure to rise again in the downstream fluid of the low pressure areas.

As the diameter ratio increased, the scope of the local high pressure areas near the rear end of the piped carriage gradually reduced, and the local high pressure areas near the front end of the piped carriage gradually increased. This occurred because with the increase of the diameter ratio, the decreasing relative velocity between the rear end of the piped carriage and the pipe fluid caused a decrease in the degree to which kinetic energy was converted into pressure energy. Likewise, the increasing relative velocity between the front end of the piped carriage and the pipe fluid increased the conversion of kinetic energy into the pressure energy. With the increase of the diameter ratio, the pressure drop in the downstream flow field of the piped carriage gradually became more severe. This occurred because increases in the diameter ratio increased the extent of back-flow areas near the front end of the piped carriage, thus causing severe energy dissipation as reflected by the increasing pressure drop. In the upstream flow field of the piped carriage, the piped carriage with the diameter ratio of 0.75 caused the minimum pressure while the piped carriage with the diameter ratio of 0.95 caused the maximum pressure. The reason for these results was that the piped carriages with the diameter ratio of 0.75 and 0.95 caused the smallest and largest energy losses, respectively. Other reasons will be described in detail in Section 6.7.

Figure 20 showed the pressure distributions of transporting the piped carriage having a diameter ratio of 0.6 at different times under the pipe discharge of $50 \mathrm{~m}^{3} \cdot \mathrm{h}^{-1}$.

As shown in Figure 20, the pressure in the near-wall of the piped carriage showed an overall decreasing trend over time. This occurred because the instantaneous speed of the piped carriage changed within a limited range, leading to the same energy losses caused by the piped carriage transport within the pipelines at different times. Due to the decreasing pressure along the pipeline, pressure gradually decreased in the nearwall of the piped carriage as the piped carriage moved towards the downstream flow field of the conveying pipe.

6.6. Vorticity Magnitude Distributions. Figure 21 showed the vorticity magnitude distributions when the piped carriage with the different diameter ratios arrived at the location that 


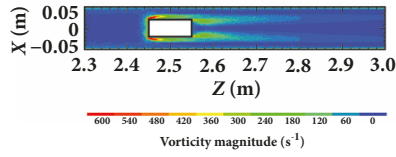

(a) $b=0.40 ; V_{b}=0.4296 \mathrm{~m} \cdot \mathrm{s}^{-1}$

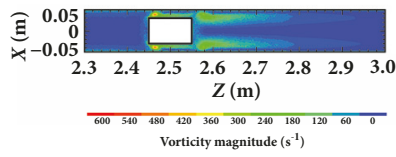

(e) $b=0.60 ; V_{b}=1.2758 \mathrm{~m} \cdot \mathrm{s}^{-1}$

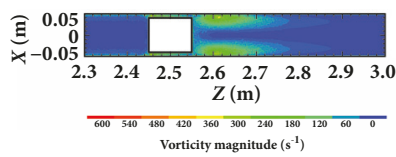

(i) $b=0.80 ; V_{b}=1.6053 \mathrm{~m} \cdot \mathrm{s}^{-1}$

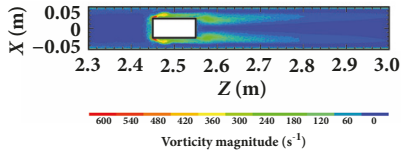

(b) $b=0.45 ; V_{b}=0.6982 \mathrm{~m} \cdot \mathrm{s}^{-1}$

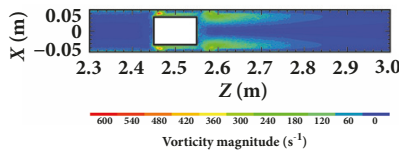

(f) $b=0.65 ; V_{b}=1.3971 \mathrm{~m} \cdot \mathrm{s}^{-1}$

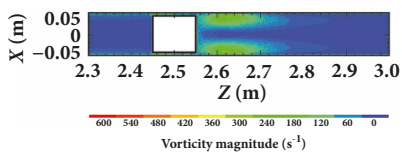

(j) $b=0.85 ; V_{b}=1.6335 \mathrm{~m} \cdot \mathrm{s}^{-1}$

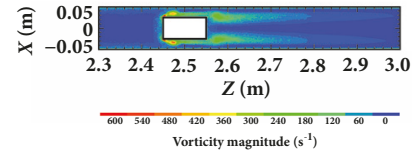

(c) $b=0.50 ; V_{b}=0.9439 \mathrm{~m} \cdot \mathrm{s}^{-1}$

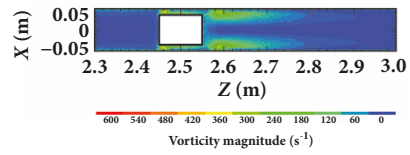

(g) $b=0.70 ; V_{b}=1.4905 \mathrm{~m} \cdot \mathrm{s}^{-1}$

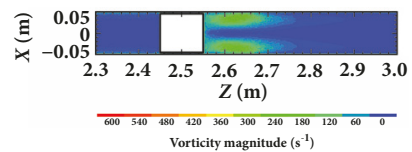

(k) $b=0.90 ; V_{b}=1.6544 \mathrm{~m} \cdot \mathrm{s}^{-1}$
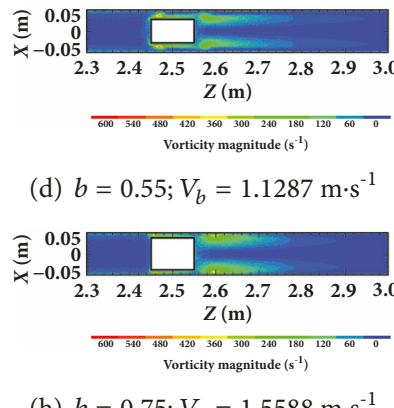

(h) $b=0.75 ; V_{b}=1.5588 \mathrm{~m} \cdot \mathrm{s}^{-1}$

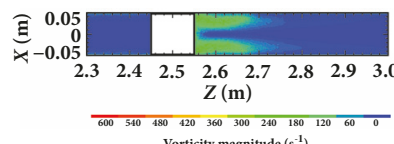

(l) $b=0.95 ; V_{b}=1.6687 \mathrm{~m} \cdot \mathrm{s}^{-1}$

FIGURE 21: Vorticity magnitude distributions of transporting a piped carriage with different diameter ratios.

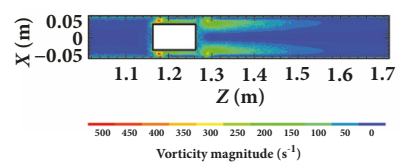

(a) $t=0.4 \mathrm{~s} ; S_{c}=1.213 \mathrm{~m} ; V_{c}=$ $1.2779 \mathrm{~m} \cdot \mathrm{s}^{-1}$

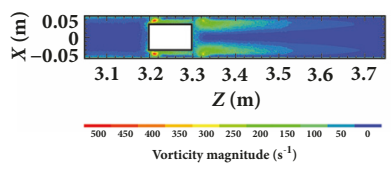

(e) $t=2.0 \mathrm{~s} ; S_{c}=3.248 \mathrm{~m} ; V_{c}=$ $1.2867 \mathrm{~m} \cdot \mathrm{s}^{-1}$

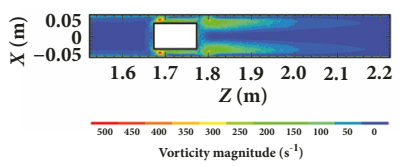

(b) $t=0.8 \mathrm{~s} ; S_{c}=1.723 \mathrm{~m} ; V_{c}=$ $1.2652 \mathrm{~m} \cdot \mathrm{s}^{-1}$

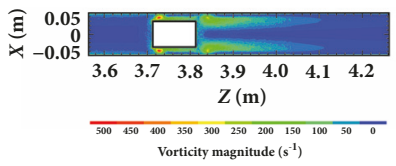

(f) $t=2.4 \mathrm{~s} ; S_{c}=3.763 \mathrm{~m} ; V_{c}=$ $1.2685 \mathrm{~m} \cdot \mathrm{s}^{-1}$

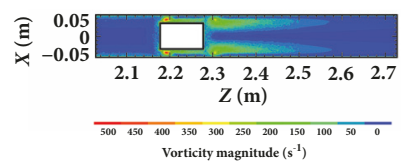

(c) $t=1.2 \mathrm{~s} ; S_{c}=2.228 \mathrm{~m} ; V_{c}=$ $1.2812 \mathrm{~m} \cdot \mathrm{s}^{-1}$

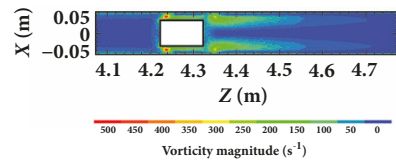

(g) $t=2.8 \mathrm{~s} ; S_{c}=4.273 \mathrm{~m} ; V_{c}=$ $1.2836 \mathrm{~m} \cdot \mathrm{s}^{-1}$

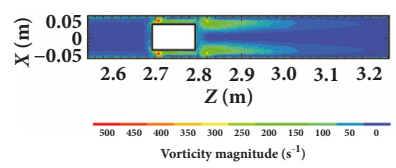

(d) $t=1.6 \mathrm{~s} ; S_{c}=2.743 \mathrm{~m} ; V_{c}=$ $1.2753 \mathrm{~m} \cdot \mathrm{s}^{-1}$

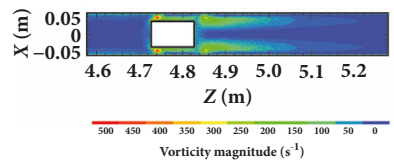

(h) $t=3.2 \mathrm{~s} ; S_{c}=4.777 \mathrm{~m} ; V_{c}=$ $1.2723 \mathrm{~m} \cdot \mathrm{s}^{-1}$

FIGURE 22: Vorticity magnitude distributions of transporting a piped carriage at different times.

was $2.5 \mathrm{~m}$ from the inlet cross-section of the test section under the pipe discharge of $50 \mathrm{~m}^{3} \cdot \mathrm{h}^{-1}$.

As shown in Figure 21, because the projection of the piped carriage onto the central horizontal cross-section of the pipeline was symmetrical around the $Z$ axis, the vorticity magnitude distributions were more symmetrical. The vorticity magnitude of the internal flow field within the pipelines was mainly distributed in the near-wall areas of the cylindrical pipe, and at the interface between the annular slit flow and the downstream fluid of the piped carriage. The boundary layer separation that formed in the near-wall areas of the cylindrical barrel caused the phenomena of a local vortex, which in turn caused the distinct vorticity magnitude distributions. In addition, the mixing process between the annular slit flow and the downstream fluid at their interface of the piped carriage mutually caused the formation of the obvious local vorticity.

The vorticity magnitude of the entrance location was larger than that at the exit location for the annular slit areas because the fluid at the rear end of the piped carriage suddenly contracted, and the annular slit flow was separated from the boundary of the piped carriage; thus, there were obvious vorticity magnitude distributions in the entrance location of the annular slit areas. As the diameter ratio increased, the vorticity magnitude in the near-wall areas of the cylindrical pipe and at the downstream flow field of the piped carriage gradually increased. The vorticity magnitude in the near-wall areas of the cylindrical pipe occurred because the affected scope of boundary layer separation increased as the diameter ratio increased. However, the vorticity magnitude at the downstream flow field of the piped carriage increased because the increasing velocity and back-flow areas caused serious blending with the downstream fluid of the piped carriage, thus producing an increase in the vorticity magnitude with the increase of the diameter ratio. With the increase of the diameter ratio, the affected areas of the vorticity magnitude near the front end of the piped carriage gradually decreased. The phenomenon occurred because the increasing diameter ratio led to an increase in the back-flow areas mainly existed the front end of the piped carriage and caused the vortex attenuation rapidly within an extremely limit range; thus producing a decrease in the affected areas of the vorticity magnitude.

Figure 22 showed the vorticity magnitude distributions of transporting a piped carriage having a diameter ratio of 0.6 at different times under the pipe discharge of $50 \mathrm{~m}^{3} \cdot \mathrm{h}^{-1}$. 


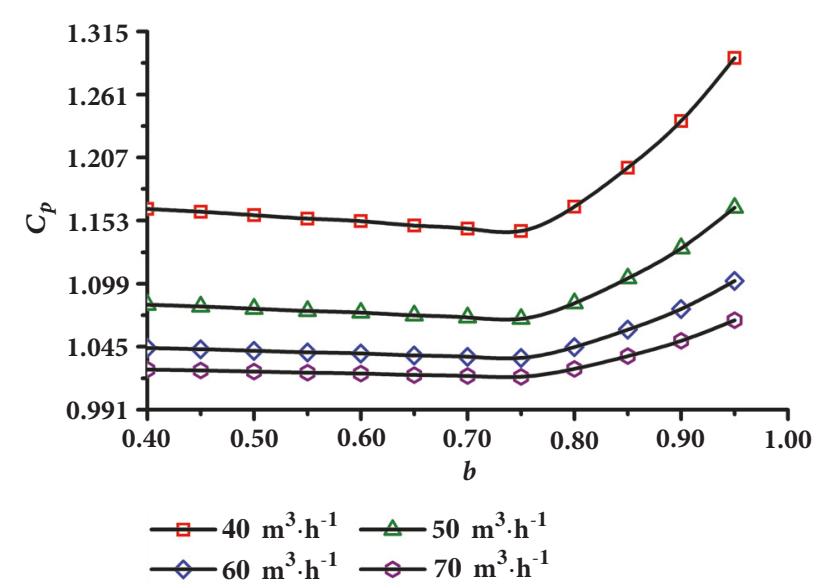

FIGURE 23: Relationship between average pressure drop coefficient and diameter ratio for a piped carriage.

Due to the interaction between the piped carriage and the pipe fluid, the vorticity magnitude distributions at the downstream flow field of the piped carriage appeared to be unsteady. However, as can be seen from Figure 22, the vorticity magnitude distributions of the horizontal section within the pipelines transporting the piped carriage at different times were the same. There was no periodic change of the vorticity magnitude distributions over time. The difference between the maximum vorticity magnitude at different times and the average maximum vorticity magnitude did less than $1.26 \%$. This appeared because the instantaneous speed of the piped carriage changed within a limited range, leading to a small change in the unsteady characteristics of the vorticity magnitude distributions of the bidirectional interaction system formed by the piped carriage and the pipe fluid at any time.

6.7. Pressure Drop Characteristics. The energy losses are analyzed using the pressure drop coefficient. The pressure drop coefficient is the ratio of the total pressure drop for the piped carriage flow to the total pressure drop that would exist if fluid alone flowed in the same pipe at the same average axial velocity. Figure 23 showed the changes in the pressure drop coefficient as the diameter ratio increased. Because the pressure drop coefficient fluctuated irregularly over time, the energy losses of the piped carriage were examined using the average pressure drop coefficient. With increasing diameter ratio, the average pressure drop coefficient first decreased and then increased, and reached its lowest value when the diameter ratio of the piped carriage was 0.75 . According to the data presented in Figure 23, when the diameter ratio was less than 0.75 , the speed of the piped carriage transport played a leading role such that increasing speed of the piped carriage decreased the local energy losses. When the diameter ratio was greater than 0.75 , the diameter of the piped carriage played a dominant role such that the local energy losses increased as the diameter of the piped carriage increased.

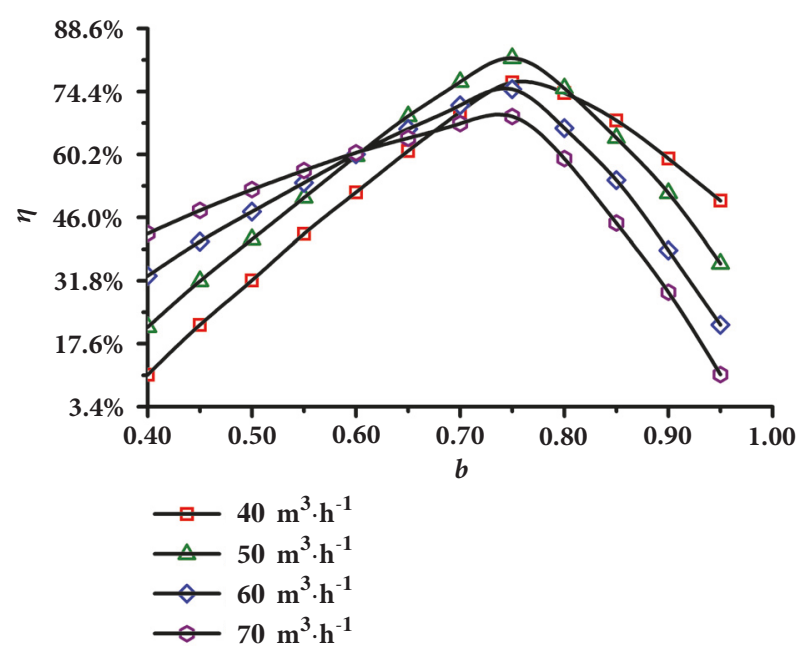

FIGURE 24: Relationship between mechanical efficiency and diameter ratio for a piped carriage.

6.8. Mechanical Efficiencies. Mechanical efficiency was an important index of the performance of mechanical properties. In physics, Mechanical efficiency denoted the transformation efficiency with which the total energy of fluid was changed into mechanical energy for the piped carriage transport. The potential energy of the fluid included mechanical energy (useful work) for the piped carriage and the internal energy of the fluid. The total energy in HCPs was the total energy losses of the fluid around the piped carriage and the mechanical energy was the kinetic energy of the piped carriage. Mechanical efficiency was determined as the ratio of the kinetic energy of the piped carriage to the total energy losses of the fluid around the piped carriage, which was written as follows:

$$
\eta=\frac{F_{d} V_{b}}{A_{p} V_{a} \rho g\left(\Delta P_{m}-\Delta P_{n}\right)}
$$

where $A_{p}$ was the pipe cross-sectional areas.

Figure 24 showed the changes in the mechanical efficiency of the piped carriage as the diameter ratio changes. It can be seen in Figure 24 that clearly the mechanical efficiency of the piped carriage first increased and then decreased as the diameter ratio increased, and it reached its maximum value $(81.3 \%)$ when the diameter ratio was 0.75 . Thus, this high efficiency indicated that HCPs had a higher transport efficiency than other transport modes, a conclusion that was consistent with the conclusion about energy losses in Section 6.7. Furthermore, the optimal diameter ratio of the piped carriage was 0.75 . When the diameter ratio was less than 0.75 , the mechanical efficiency of the piped carriage increased with the increase of the pipe discharges, and when the diameter ratio was greater than 0.75 , the mechanical efficiency gradually decreased with the increase of the pipe discharges. This appeared because when the diameter ratio was less than 0.75 , the increasing rate of the energy losses of the fluid around the piped carriage was gradually less than that of the average speed of the piped carriage, and when 

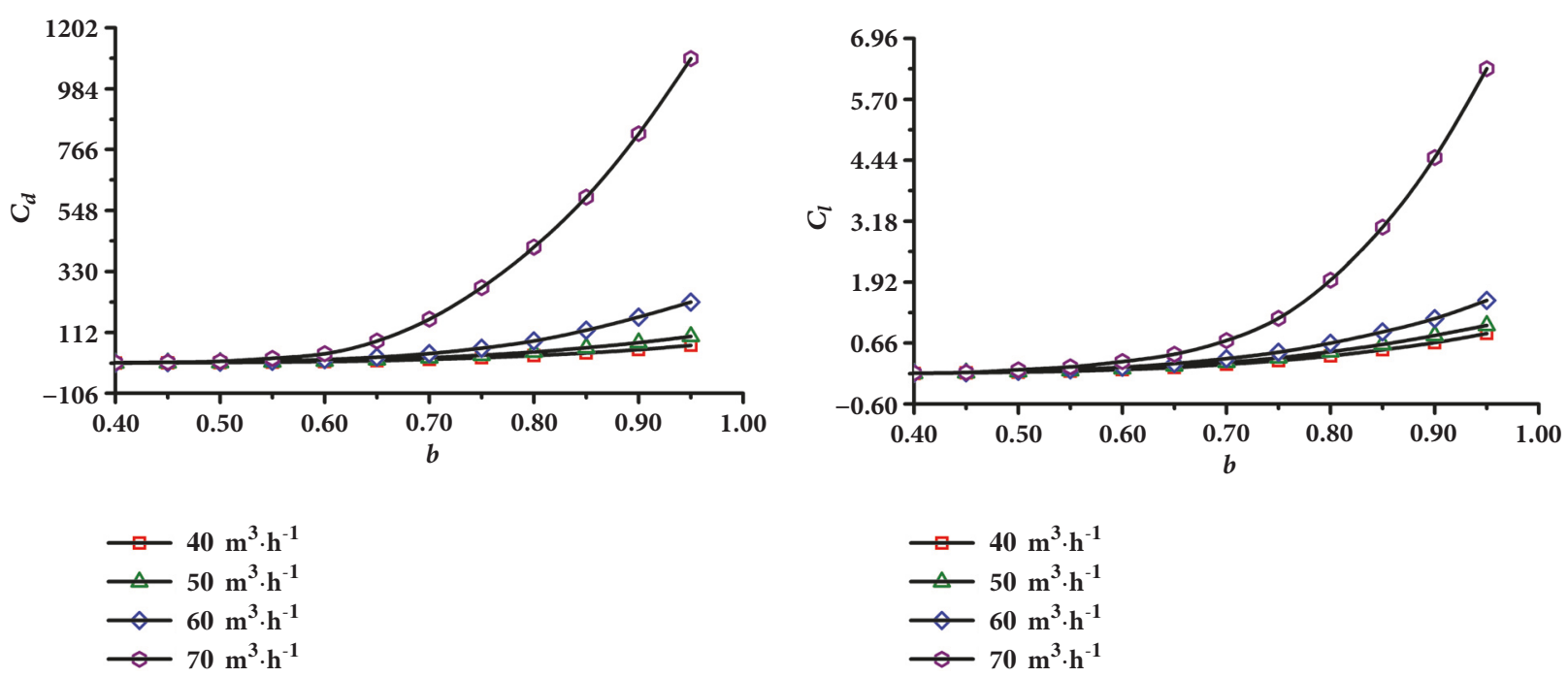

(a) Relationship between $C_{d}$ and $b$

(b) Relationship between $C_{l}$ and $b$

FIGURE 25: Relationship between average force coefficient and diameter ratio for a piped carriage.

the diameter ratio was greater than 0.75 , it was contrary. According to Figure 24, the optimal combination of the mechanical efficiency for HCPs was 0.75 in diameter and 50 $\mathrm{m}^{3} \cdot \mathrm{h}^{-1}$ in pipe discharge, the above conclusions were suitable for the case in which the length of the piped carriage was 0.1 $\mathrm{m}$ and the transport loading was $0.6 \mathrm{~kg}$.

6.9. Force Statistics. Figure 25 showed the changing trends of both the average drag coefficient and the average lift coefficient of the piped carriage as a function of changes in the diameter ratio. The drag coefficient was the ratio of the drag of the piped carriage to the product of both the hydrodynamic pressure and the reference areas that were perpendicular to flow. Because the drag coefficient of the piped carriage fluctuated over time, the drag characteristic of the piped carriage was analyzed using the average drag coefficient. As shown in Figure 25(a), the average drag coefficient of the piped carriage increased exponentially as the diameter ratio increased. This occurred because the diameter ratio increased; the increasing rate of dynamic pressure was larger than the decreasing rates of the reference areas; consequently, the product of the two parameters showed a decreasing tendency. Due to the smaller extent of variation in the drag for the piped carriage with different diameter ratios, the average drag coefficient increased exponentially.

The asymmetric structure of the upper and the lower surfaces of the piped carriage generated a lifting action. The lift coefficient referred to the ratio of the lift force of the piped carriage to the product of both the hydrodynamic pressure and the reference areas that are parallel to flow. Because the lift coefficient of the piped carriage fluctuated with time, the lift characteristic of the piped carriage was analyzed using the average lift coefficient. As shown in Figure 25(b), the direction of the lift was directly vertical (perpendicular to flow). This occurred because the path length of the upper surface was far greater than that of the lower surface of the support body, which caused the pressure on the upper surface to be less than the pressure on the lower surface. As the diameter ratio increased, the average lift coefficient of the piped carriage increased exponentially because the growth rates of the dynamic pressure were much greater than the rates at which the reference areas decreased, giving the product of the two parameters a decreasing tendency. Hence, the average lift coefficient showed an exponential increase trend gradually.

\section{An Optimization Model of HCPs}

Optimization of HCPs was of great significance for its commercial promotion of the piped carriage transport. An optimization model of HCPs has already been developed by the author in a previous published literature $[12,37]$. Published literature was mainly limited to a cylindrical capsule and a rectangular capsule with density equal to that of its carrier fluid. In this paper, the optimization model of HCPs transporting a piped carriage within the horizontal pipelines was established for the first time, which was designed based on the Least-Cost Principle; i.e., the pipeline transporting capsules was designed such that the total cost of the pipeline was minimum. The total cost of a pipeline transporting piped carriage consists of the manufacturing cost of the pipeline and the piped carriage plus the operating cost of the system.

$$
R_{t}=R_{m}+R_{o}
$$

where $R_{m}$ was the manufacturing cost of the pipeline and the piped carriage; $R_{o}$ was the cost of the operating cost of the system.

The manufacturing cost can be further divided into the cost of the pipeline and the cost of the piped carriage. The operating cost referred to the cost of the power being consumed. Therefore, the total cost of HCPs consisted of the 
cost of the pipe, the cost of the piped carriage and the cost of power. It can be written as follows:

$$
R_{t}=R_{a}+R_{b}+R_{p}
$$

where $R_{t}$ was the total cost of HCPs; $R_{a}$ was the cost of the pipeline; $R_{b}$ was the cost of the piped carriage; $R_{p}$ was the cost of power.

7.1. Cost of Pipe. The cost of the pipe per unit weight of the pipe material was written as follows [35]:

$$
R_{a}=C_{1} \pi D_{p} e_{p} \rho_{p} g L_{p}
$$

where $e_{p}$ was the thickness of the pipe wall; $\rho_{p}$ was the density of the pipe; $L_{p}$ was the length of the pipe; $C_{1}$ was the cost of pipe per unit of weight.

7.2. Cost of Piped Carriage. The cost of the piped carriage per unit weight of the piped carriage material was expressed as follows:

$$
R_{b}=C_{2} \pi k D_{p} L_{c} e_{c} \rho_{c} g
$$

where $C_{2}$ was the cost of the piped carriage per unit of weight; $e_{c}$ was the thickness of the piped carriage wall; $L_{c}$ was the length of the piped carriage; $\rho_{c}$ was the mixture densities.

7.3. Cost of Power. The cost of power was expressed as follows [12]:

$$
R_{p}=C_{3} t_{p} P_{w}=\frac{C_{3} t_{p} Q_{m} \Delta P_{\text {total }}}{3.6 \times 10^{5} \eta_{p}}
$$

where $P_{w}$ was the power requirement of the pipeline transporting piped carriage; $C_{3}$ was the cost of power per kilowatt hour; $\eta_{p}$ was the efficiency of centrifugal water pump, generally the efficiency of industrial pumping unit ranges between 60 to $75 \%$. $t_{p}$ was the run time for the centrifugal water pump; $\Delta P_{\text {total }}$ was the total pressure drop of the flow of the piped carriage; $Q_{m}$ was the mixture pipe discharge. Taking into account the service life of the centrifugal water pump, its run time was generally calculated for a one-year period.

HCPs was a typical solid-liquid two-phase flow. To this end, Liu [53] reported the expression to solve the mixture pipe discharge, which was written as:

$$
Q_{m}=0.25 \pi D_{p}^{2} V_{a}
$$

The above formula only was suitable for a circular pipe.

Asim [35, 36] used the existing formulas to calculate total pressure drop of a cylindrical capsule and a rectangular capsule. The total pressure drop of the piped carriage was influenced by several factors such as rolling frictional resistance coefficient, transport loading and support body. At present, there was no appropriate formula for the total pressure drop, which can only be investigated by numerical simulation. From this analysis, studies on an optimization model of HCPs transporting the piped carriage within the pipelines had great significances.
7.4. Optimization Methods. The optimization model of HCPs was put forward was to be in order to seek the lowest construction cost. The lowest construction cost was influenced by several factors such as the diameter of the piped carriage, length of the piped carriage, diameter of the pipeline and transport load. The length of the piped carriage was set to 0.1 $\mathrm{m}$ due to limitation of the bending. The transport loading was mainly limited by the internal volume of the piped carriage, so it in this paper was set to $0.6 \mathrm{~kg}$. According to the studies on the optimal diameter ratio of piped carriage in Section 6, it can be seen that the piped carriage having the diameter ratio of 0.75 had the lowest total pressure drop. Therefore, the model of HCPs can be optimized simply by determining the diameter of the conveying pipes.

The method for determining the diameter of the conveying pipe comprised the following steps:

(1) Assume a value of the pipe diameter $D_{p}$.

(2) The length of the pipeline was obtained through the dropping and receiving position of the piped carriage.

(3) Calculate the cost of pipes and a piped carriage based on the materials for the pipelines and the piped carriage and the market prices of these materials (see (33) and (34)).

(4) According to the conclusion mentioned in Section 6, the diameter ratio of the piped carriage was determined.

(5) Confirm the diameter of the piped carriage $D_{e}$.

(6) Assume the efficiency of the centrifugal water pump.

(7) Combined with the corresponding dimensions of HCPs, the total pressure drop of the piped carriage transport was calculated using the bidirectional fluid-structure interaction method.

(8) Assume the mixture pipe discharge $Q_{m}$.

(9) Calculate the cost of power (see (35) and (36)).

(10) Calculate the total cost of HCPs (see (31) and (32)).

(11) Find out the optimal diameter of the conveying pipe by comparing the total cost of HCPs under different pipe diameters.

7.5. Design Example. According to Section 6, the energy losses of transporting the piped carriage with a diameter ratio of 0.75 was the lowest within the horizontal pipeline. For this reasons, the piped carriage with the diameter ratio of 0.75 was studied. The optimization model of HCPs was used to find out the optimal diameter of the conveying pipe and the pumping power required.

Solution: according to the current market, the values of $C_{1}, C_{2}$, and $C_{3}$ are $10.8 ¥ / \mathrm{N}, 12.6 ¥ / \mathrm{N}$, and $0.9 ¥ /(\mathrm{kW} \bullet \mathrm{h})$ (industrial electricity), respectively. The efficiency of the centrifugal water pump was assumed to be $70 \%$. The pipe was $28.26 \mathrm{~m}$ long with a thickness of $5 \mathrm{~mm}$ and a pipe discharge of $50 \mathrm{~m}^{3} \cdot \mathrm{h}^{-1}$. In addition, the piped carriage was $0.1 \mathrm{~m}$ long with a thickness of $5 \mathrm{~mm}$, and the transport loading of the piped carriage was $0.6 \mathrm{~kg}$. The rolling frictional resistance coefficient between the piped carriage wall and the Plexiglas pipe wall was 0.462 from experiments. The bidirectional fluid-structure interaction model was used to obtain the total pressure drop of transporting the piped carriage within the horizontal pipelines. According to the optimization steps mentioned in Section 7.4, the following results have been obtained, as shown in Table 3. It is noteworthy that the cost 
TABLE 3: Relationship between different costs of HCPs and pipe diameter.

\begin{tabular}{lccccccccc}
\hline$D_{p} / \mathrm{mm}$ & 60 & 70 & 80 & 90 & 100 & 110 & 120 & 130 & 140 \\
\hline$R_{a} / ¥$ & 3395.643 & 3961.583 & 4527.524 & 5093.464 & 5659.405 & 6225.345 & 6791.286 & 7357.226 & 7923.166 \\
$R_{b} / ¥$ & 92.827 & 108.299 & 123.770 & 139.241 & 154.712 & 170.184 & 185.655 & 201.126 & 216.597 \\
$R_{p} / ¥$ & 11807.058 & 6435.096 & 3808.443 & 2400.259 & 1589.682 & 1095.932 & 780.973 & 572.213 & 429.292 \\
$R_{t} / ¥$ & 15295.528 & 10504.977 & 8459.737 & 7632.964 & 7403.799 & 7491.460 & 7757.913 & 8130.565 & 8569.056 \\
$P_{w} / \mathrm{kW}$ & 1.5183 & 0.8275 & 0.4897 & 0.3086 & 0.2044 & 0.1409 & 0.1004 & 0.0735 & 0.0552 \\
\hline
\end{tabular}

of the piped carriage and the pipelines was a one-off cost, whereas the electricity cost was an annual cost.

The results presented in Table 3 depicted that the optimal diameter was $100 \mathrm{~mm}$ because the total cost of HCPs was minimum at $D_{p}=100 \mathrm{~mm}$, which further verified that it was reasonable to use the pipes with the diameter of $100 \mathrm{~mm}$ for this study. Corresponding to the optimal diameter of the pipeline, the power of the pumping unit required was $02044 \mathrm{~kW}$. It can be further seen that as the pipe diameter increased, the cost of both the pipelines and the piped carriage increased. This was due to the fact that pipes of larger diameters were more expensive than pipes of relatively smaller diameter. Moreover, as the pipe diameter increased, the cost of power decreased. This was due to fact that for the same flow velocity of the pipe fluid, the increasing of the pipe diameter led to the decreasing of the flow velocity, resulting in a drastic reduction of the energy losses within the pipelines. The cost of power was proportional to the flow velocity of the pipeline; hence, increase in the pipe diameter decreased the cost of power.

\section{Conclusion}

The hydraulic characteristics of piped carriage transport under different diameter ratios within the horizontal pipelines were analyzed using the model experiment and the numerical simulation. The simulated results were in good agreement with the experimental results, which further indicated that the bidirectional fluid-structure interaction numerical simulation had apparent advantages for the analysis of HCPs compared with the unidirectional fluidstructure interaction methods. The main conclusions are summarized as follows:

(1) A logarithmic growth relationship exists between the diameter ratio and the average speed of the piped carriage with density larger than that of its carrier fluid.

(2) As the pipe diameter ratio increases, the affected areas of axial velocity, radial velocity, and pressure gradually increase, the affected areas of the vorticity magnitude gradually decrease, and the affected areas of circumferential velocity gradually decrease first and then increase near the front end of the piped carriage. With the increase of the diameter ratio, the average drag coefficient and the average lift coefficient of the piped carriage increase exponentially, and the piped carriage experiences upward lift under arbitrary diameter ratios.

(3) The combined effects of energy dissipation and energy conversion cause local low pressure areas to develop near the front end of the piped carriage, but energy conversion causes the downstream pressure of the piped carriage to subsequently increase. As the diameter ratio increases, the average pressure drop coefficient of the piped carriage first decreases and then increases, while the mechanical efficiency of the piped carriage first increases and then decreases.

(4) When piped carriage moves through the pipeline, the axial velocity distributions, the radial velocity distributions, the circumferential velocity distributions, and the vorticity magnitude distributions in the near-wall areas of the piped carriage inside the pipeline are basically the same, while the pressure distributions show a decreased trend.

(5) Based on Least-Cost Principle, the optimization model of HCPs can output the optimal pipe diameter. A practical example has been included in order to verify the usage and effectiveness of this optimization model.

\section{Data Availability}

The data used to support the findings of this study are available from the corresponding author upon request.

\section{Conflicts of Interest}

The authors declare that there are no conflicts of interest regarding the publication of this paper.

\section{Acknowledgments}

Supports from the Collaborative Innovation Center of New Technology of Water-Saving and Secure and Efficient Operation of Long-Distance Water Transfer Project at Taiyuan University of Technology, Shanxi Province Supercomputing and Data Center, and Shanxi Province Scientific Cloud Computing Center are acknowledged. This project is supported by the National Natural Science Foundation of China (Grants nos. 51179116, 51109155, and 50579044) and the Natural Science Foundation of Shanxi Province (Grants nos. 2015011067 and 201701D221137).

\section{References}

[1] R. A. S. Brown, "Capsule pipeline research at the alberta research council, 1958-1978," Journal of pipelines, vol. 6, no. 1, pp. 75-82, 1987.

[2] P. K. Swamee, "Design of pipelines to transport neutrally buoyant capsules," Journal of Hydraulic Engineering, vol. 124, no. 11, pp. 1155-1160, 1998.

[3] M. E. Charles, "The pipeline flow of capsules: Part 2: Theoretical analysis of the concentric flow of cylindrical forms," The 
Canadian Journal of Chemical Engineering, vol. 41, no. 2, pp. 4651, 1963.

[4] R. Newton, P. J. Redberger, and G. F. Round, "The pipeline flow of capsules part 6 - numerical analysis of some variables determining free flow," The Canadian Journal of Chemical Engineering, vol. 42, no. 4, pp. 168-173, 1964.

[5] H. S. Ellis, "The pipeline flow of capsules: Part 3 - An experimental investigation of the transport by water of single cylindrical and spherical capsules with density equal to that of the water," The Canadian Journal of Chemical Engineering, vol. 42, no. 1, pp. $1-8,1964$.

[6] J. Kruyer and W. T. Snyder, "Relationship between capsule pulling force and pressure gradient in a pipe," The Canadian Journal of Chemical Engineering, vol. 53, no. 4, pp. 378-383, 1975.

[7] B. Latto and K. W. Chow, "Hydrodynamic transport of cylindrical capsules in a vertical pipeline," The Canadian Journal of Chemical Engineering, vol. 60, no. 6, pp. 713-722, 1982.

[8] Y. Tomita, M. Yamamoto, and K. Funatsu, "Motion of a single capsule in a hydraulic pipeline," Journal of Fluid Mechanics, vol. 171, pp. 495-508, 1986.

[9] Y. Fujiwara, Y. Tomita, H. Satou, and K. Funatsu, "Characteristics of hydraulic capsule transport," JSME International Journal Series B Fluids and Thermal Engineering, vol. 37, no. 1, pp. 89-95, 1994.

[10] C. W. Lenau and M. M. El-Bayya, "Unsteady flow in hydraulic capsule pipeline," Journal of Engineering Mechanics, vol. 122, no. 12, pp. 1168-1173, 1996.

[11] K. Yanaida and M. Tanaka, "Drag coefficient of a capsule inside a vertical angular pipe," Powder Technology, vol. 94, no. 3, pp. 239-243, 1997.

[12] V. C. Agarwal and R. Mishra, "Optimal design of a multi-stage capsule handling multi-phase pipeline," International Journal of Pressure Vessels and Piping, vol. 75, no. 1, pp. 27-35, 1998.

[13] L. Y. Hwang, D. J. Wood, and D. T. Kao, "Capsule hoist system for vertical transport of coal and other mineral solids," The Canadian Journal of Chemical Engineering, vol. 59, no. 3, pp. 317324, 1981.

[14] H. G. Polderman, "Design rules for hydraulic capsule transport systems," Journal of Pipelines, vol. 3, no. 2, pp. 123-136, 1982.

[15] M. Tachibana and Y. Matsumoto, "Basic studies on hydraulic capsule transportation: Part 1 . Loss due to cylindrical capsules set in circular pipe flow," Bulletin of Jsme, vol. 24, no. 192, pp. 987-994, 1981.

[16] R. Mishra, S. N. Singh, and V. Seshadri, "Improved model for the prediction of pressure drop and velocity field in multisized particulate slurry flow through horizontal pipes," Powder Handling and Processing, vol. 10, no. 3, pp. 279-287, 1998.

[17] P. Vlasak and V. Berman, "A contribution to hydrotransport of capsules in bend and inclined pipeline sections," Handbook of Powder Technology, vol. 10, pp. 521-529, 2001.

[18] H. S. Ellis and J. Kruyer, "Minimizing the pressure gradients in capsule pipelines," The Canadian Journal of Chemical Engineering, vol. 52 , no. 4 , pp. 457-462, 1974.

[19] I. Teke and D. Ulusarslan, "Mathematical expression of pressure gradient in the flow of spherical capsules less dense than water," International Journal of Multiphase Flow, vol. 33, no. 6, pp. 658674, 2007.

[20] D. Ulusarslan, "Comparison of experimental pressure gradient and experimental relationships for the low density spherical capsule train with slurry flow relationships," Powder Technology, vol. 185, no. 2, pp. 170-175, 2008.
[21] D. Barthès-Biesel, "Modeling the motion of capsules in flow," Current Opinion in Colloid \& Interface Science, vol. 16, no. 1, pp. 3-12, 2011.

[22] D. Ulusarslan, "Experimental investigation of the effect of diameter ratio on velocity ratio and pressure gradient for the spherical capsule train flow," European Journal of Mechanics B/Fluids, vol. 37, pp. 42-47, 2013.

[23] C. Dupont, P. Le Tallec, D. Barthès-Biesel, M. Vidrascu, and A.-V. Salsac, "Dynamics of a spherical capsule in a planar hyperbolic flow: Influence of bending resistance," Procedia IUTAM, vol. 16, pp. 70-79, 2015.

[24] R. G. Garner and G. D. Raithby, "Laminar flow between a circular tube and a cylindrical eccentric capsule," The Canadian Journal of Chemical Engineering, vol. 56, no. 2, pp. 176-180, 1978.

[25] U. Kumar, R. Mishra, S. N. Singh, and V. Seshadri, "Effect of particle gradation on flow characteristics of ash disposal pipelines," Powder Technology, vol. 132, no. 1, pp. 39-51, 2003.

[26] N. C. G. Markatos, R. Sala, and D. R. Spalding, "Flow in an annulus of non-uniform gap," Transactions of the Institution of Chemical Engineers, vol. 56, pp. 28-35, 1978.

[27] V. K. Garg, "Capsule-pipelining-an improved theoretical analysis," Journal of Fluids Engineering, vol. 99, no. 4, pp. 763-771, 1977.

[28] K. Ogawa, S. Ito, and C. Kuroda, "Laminar-turbulent velocity profile transition for flows in concentric annuli, parallel plates and pipes," Journal of Chemical Engineering of Japan, vol. 13, no. 3, pp. 183-188, 1980.

[29] H. H. Van Den Kroonenberg, "A mathematical model for concentric horizontal capsule transport," The Canadian Journal of Chemical Engineering, vol. 56, no. 5, pp. 538-543, 1978.

[30] I. Sud and J. B. Chaddock, "Drag calculations for vehicles in very long tubes from turbulent flow theory," Journal of Fluids Engineering, vol. 103, no. 2, pp. 361-366, 1981.

[31] Y. Tomita and Y. Fujiwara, "Capsule velocity in pipelines," JSME International Journal, Series 2: Fluids Engineering, Heat Transfer, Power, Combustion, Thermophysical Properties, vol. 35, no. 4, pp. 513-518, 1992.

[32] H. G. Polderman, G. Velraeds, and W. Knol, "Turbulent lubrication flow in an annular channel," Journal of Fluids Engineering, vol. 108, no. 2, pp. 185-192, 1986.

[33] S.-B. Huang and Z.-M. Li, "Heat transfer of Bingham fluid in a concentric annulus with an axially moving inner pipe," Journal of China University of Petroleum (Edition of Natural Science), vol. 31, no. 3, pp. 94-99, 2007.

[34] M. F. Khalil, S. Z. Kassab, I. G. Adam, and M. A. Samaha, "Turbulent flow around single concentric long capsule in a pipe," Applied Mathematical Modelling: Simulation and Computation for Engineering and Environmental Systems, vol. 34, no. 8, pp. 2000-2017, 2010.

[35] T. Asim, R. Mishra, S. Abushaala, and A. Jain, "Development of a design methodology for hydraulic pipelines carrying rectangular capsules," International Journal of Pressure Vessels and Piping, vol. 146, pp. 111-128, 2016.

[36] T. Asim and R. Mishra, "Computational fluid dynamics based optimal design of hydraulic capsule pipelines transporting cylindrical capsules," Powder Technology, vol. 295, pp. 180-201, 2016.

[37] Z. Wang, Y. Sui, A.-V. Salsac, D. Barthès-Biesel, and W. Wang, "Motion of a spherical capsule in branched tube flow with finite inertia," Journal of Fluid Mechanics, vol. 806, pp. 603-626, 2016. 
[38] J. Feng, P. Y. Huang, and D. D. Joseph, "Dynamic simulation of the motion of capsules in pipelines," Journal of Fluid Mechanics, vol. 286, pp. 201-227, 1995.

[39] X. H. Sun, Y. Y. Li, and Q. F. Yan, "Experimental study on starting conditions of the hydraulic transportation on the piped carriage," in Proceedings of the 20th national conference on hydrodynamics, Beijing, China, 2007.

[40] Y. Li, X. Sun, F. Li, and R. Wang, "Hydraulic characteristics of transportation of different piped carriages in pipe," Journal of Drainage and Irrigation Machinery Engineering, vol. 28, no. 2, pp. 174-178, 2010.

[41] R. Wang, X. Sun, and Y. Li, "Transportation characteristics of piped carriage with different Reynolds numbers," Journal of Drainage and Irrigation Machinery Engineering, vol. 29, no. 4, pp. 343-358, 2011.

[42] C. Zhang, X. Sun, Y. Li, and X. Zhang, "Numerical simulation of hydraulic characteristics of cyclical slit flow with moving boundary of tube-contained raw materials pipelines hydraulic transportation," Transactions of the Chinese Society of Agricultural Engineering (Transactions of the CSAE), vol. 33, no. 19, pp. 76-85, 2017.

[43] Y. Li, X. Sun, F. Li, and J. Zhang, "Cyclical slit flow of concentricity under the moving boundary condition," Nongye Jixie Xuebao/Transactions of the Chinese Society of Agricultural Machinery, vol. 43, no. 3, pp. 230-234, 2012.

[44] X.-L. Zhang, X.-H. Sun, and Y.-Y. Li, “3-D numerical investigation of the wall-bounded concentric annulus flow around a cylindrical body with a special array of cylinders," Journal of Hydrodynamics, vol. 27, no. 1, pp. 120-130, 2015.

[45] Y. H. Jing, X. H. Guo, X. H. Sun, Y. Y. Li, and X. Y. Song, "Hydraulic characteristics of cyclical slit flow for piped carriage," Water Resources and Power, vol. 32, no. 7, pp. 151-155, 2014.

[46] X. M. Guo, Turbulence Characteristics of Hydraulic Pipeline Transportation under Different Load in Straight And Horizonal Pipeline, [Master Thesis], Taiyuan University of Technology, China, 2015.

[47] X. L. Tang, M. D. Zou, F. J. Wang, X. Q. Li, and X. Y. Shi, "Comprehensive Numerical Investigations of Unsteady Internal Flows and Cavitation Characteristics in Double-Suction Centrifugal Pump," Mathematical Problems in Engineering, vol. 2017, Article ID 5013826, 13 pages, 2017.

[48] L.-K. Song, J. Wen, C.-W. Fei, and G.-C. Bai, "Distributed collaborative probabilistic design of multi-failure structure with fluid-structure interaction using fuzzy neural network of regression," Mechanical Systems and Signal Processing, vol. 104, pp. 72-86, 2018.

[49] T. Asim, Computational Fluid Dynamics Based Diagnostics and Optimal Design of Hydraulic Capsule Pipelines [Ph.D. thesis], University of Huddersfield, 2013.

[50] V. C. Agarwal, M. K. Singh, and R. Mathur, "Empirical relation for the effect of the shape of the capsules and the end shape on the velocity ratio of heavy density capsules in a hydraulic pipeline," ARCHIVE Proceedings of the Institution of Mechanical Engineers Part E Journal of Process Mechanical Engineering 19891996, vol. 215, no. 2, pp. 147-155, 2001.

[51] S. Kosugi, "Effect of traveling resistance factor on pneumatic capsule pipeline system," Powder Technology, vol. 104, no. 3, pp. 227-232, 1999.

[52] D. Ulusarslan and I. Teke, "An experimental investigation of the capsule velocity, concentration rate and the spacing between the capsules for spherical capsule train flow in a horizontal circular pipe," Powder Technology, vol. 159, no. 1, pp. 27-34, 2005.

[53] H. Liu and K. H. Rhee, "Behavior of non-uniform-density capsules in HCP," Journal of Pipelines, vol. 6, no. 3, pp. 307-318, 1987.

[54] S. Zheng, W. Zhang, and X. Lv, "Numerical simulation of crossflow around three equal diameter cylinders in an equilateraltriangular configuration at low Reynolds numbers," Computers \& Fluids, vol. 130, pp. 94-108, 2016.

[55] C. J. Chen, X. Zhao, and Y. Y. Zhang, "A Posteriori Error Estimate for Finite Volume Element Method of the Second-Order Hyperbolic Equations," Mathematical Problems in Engineering, vol. 2015, Article ID 510241, 11 pages, 2015.

[56] W. Zhang, J. Ma, P. Wang, Z. Wang, F. Shi, and H. Liu, "Investigations on the interfacial capacitance and the diffusion boundary layer thickness of ion exchange membrane using electrochemical impedance spectroscopy," Journal of Membrane Science, vol. 502, pp. 37-47, 2016.

[57] P. Kundu, V. Kumar, Y. Hoarau, and I. M. Mishra, "Numerical simulation and analysis of fluid flow hydrodynamics through a structured array of circular cylinders forming porous medium," Applied Mathematical Modelling: Simulation and Computation for Engineering and Environmental Systems, vol. 40, no. 23-24, pp. 9848-9871, 2016.

[58] Y. P. Ma, M. X. Yi, L. F. Wang, and J. Huang, "Computation of aerodynamic noise radiated from ducted tail rotor using boundary element method," Mathematical Problems in Engineering, vol. 2017, Article ID 5726253, 13 pages, 2017.

[59] K. Yang, P. Sun, L. Wang, J. Xu, and L. Zhang, "Modeling and simulations for fluid and rotating structure interactions," Computer Methods Applied Mechanics and Engineering, vol. 311, pp. 788-814, 2016.

[60] S. M. Mehdi, M. Akhtar, A. Hussain, D. S. Alothmany, and S. Aziz, "CFD study of liquid sodium inside a wavy tube for laminar convectors: effect of reynolds number, wave pitch, and wave amplitude," Mathematical Problems in Engineering, vol. 2016, Article ID 6146195, 17 pages, 2016.

[61] X. H. Zhao, Design and Simulation for The Separation of a Taper Nose Cap from A Cruise Missile [Master Thesis], National University of Defense Technology, China, 2009.

[62] Y. He, A. E. Bayly, and A. Hassanpour, "Coupling CFD-DEM with dynamic meshing: A new approach for fluid-structure interaction in particle-fluid flows," Powder Technology, vol. 325, pp. 620-631, 2018. 


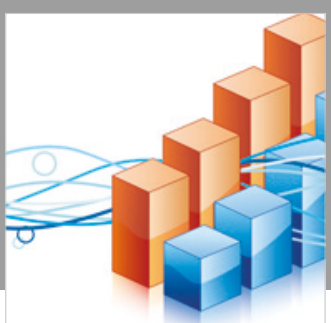

Advances in

Operations Research

\section{-n-m}
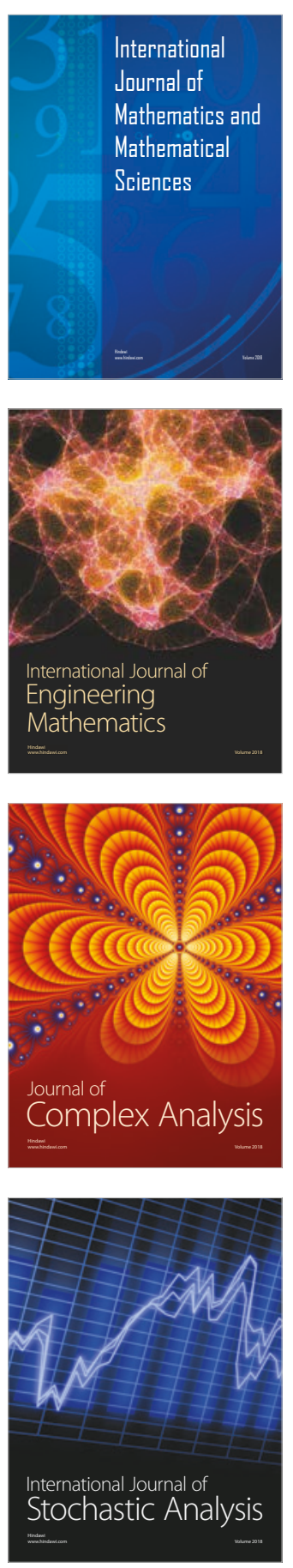
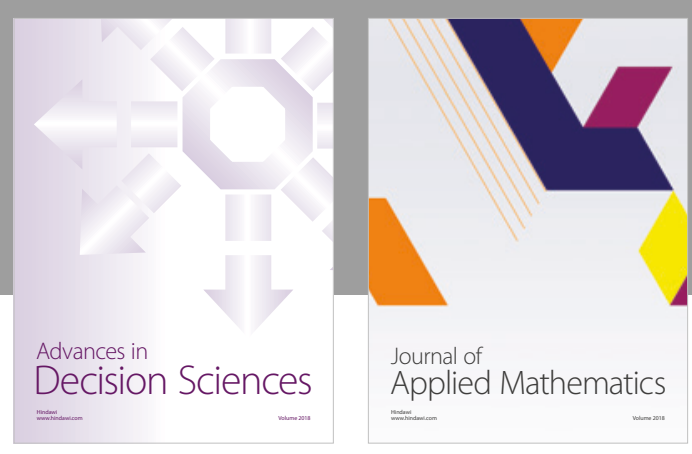

Journal of

Applied Mathematics
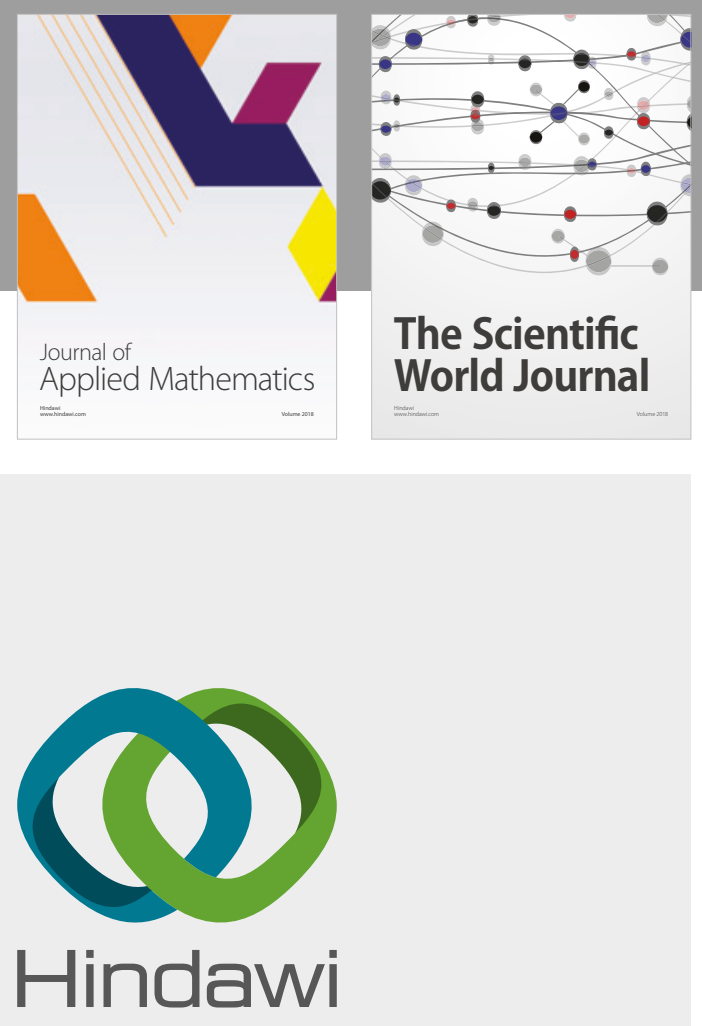

Submit your manuscripts at

www.hindawi.com

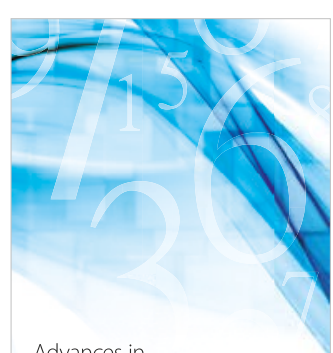

Advances in
Numerical Analysis
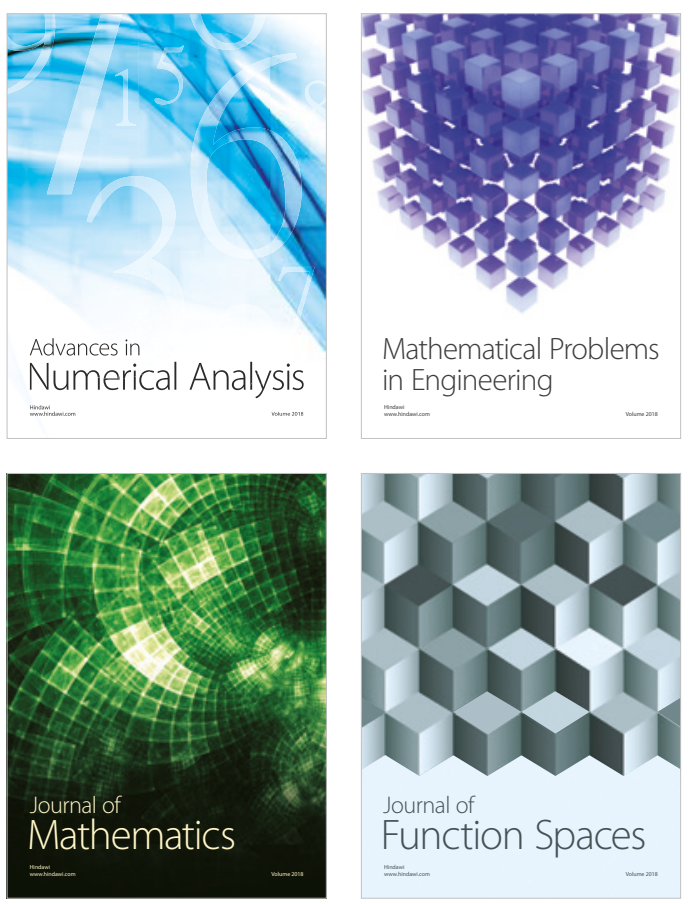

Mathematical Problems in Engineering

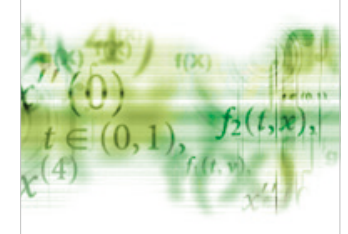

International Journal of

Differential Equations

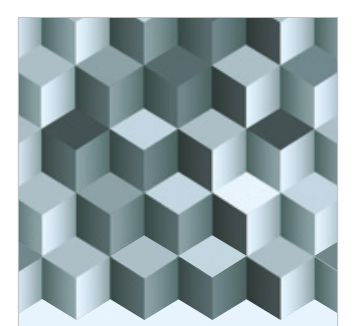

Journal of

Function Spaces

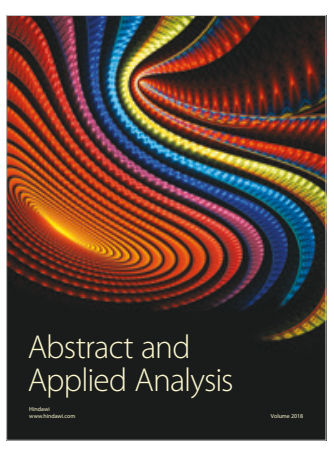

The Scientific

World Journal

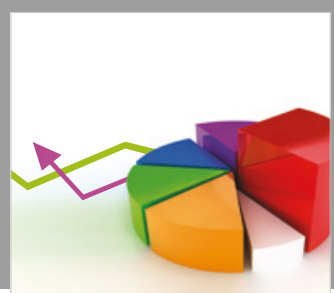

Journal of

Probability and Statistics
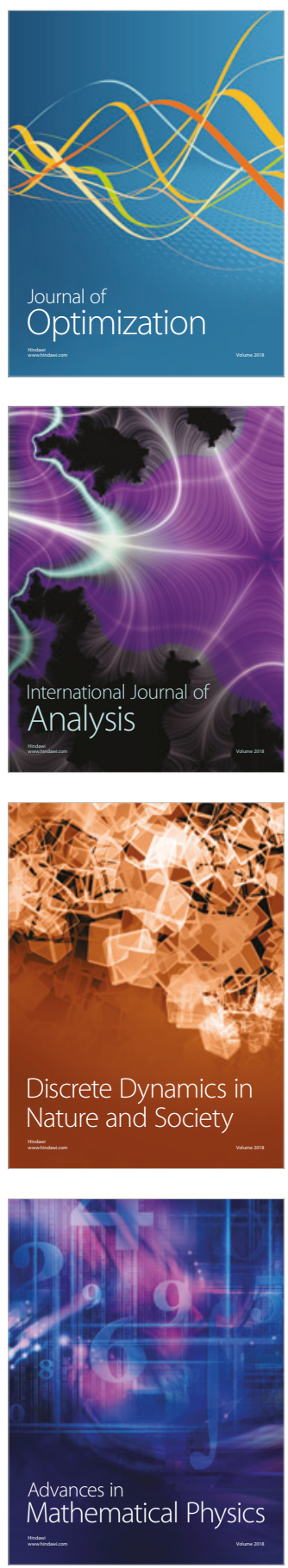